\title{
Índices biológicos fluviales en espacios naturales protegidos: ¿son adecuadas las clases de calidad de uso general?
}

\author{
Ángel Rubio-Romero* e Ignacio Granados
}

Centro de Investigación, Seguimiento y Evaluación. Parque Nacional de la Sierra de Guadarrama. Ctra. M-604 km 28,0. 28740 Rascafría (Madrid). Spain.

* Corresponding author: angel.rubio@pnsg.es

Received: 28/03/18 Accepted: 18/01/19

\begin{abstract}
RESUMEN
Índices biológicos fluviales en Espacios Naturales Protegidos: ¿son adecuadas las clases de calidad de uso general?
\end{abstract}

El Plan Director de Parques Nacionales especifica que el seguimiento del estado ecológico debe realizarse de acuerdo a los parámetros de la Directiva Marco del Agua (DMA). Sin embargo, los Parques Nacionales de montaña se caracterizan por poseer pequeñas cuencas $\left(<10 \mathrm{~km}^{2}\right)$ que quedan fuera del ámbito de la DMA. El objetivo de este artículo es evaluar si los índices bióticos utilizados habitualmente por la DMA son adecuados para evaluar el estado ecológico de cuencas pequeñas y generalmente bien conservadas en espacios protegidos de montaña. Para ello, se presentan los resultados del seguimiento del estado ecológico de los principales ríos en el ámbito del Parque Nacional de la Sierra de Guadarrama. Se ha evaluado la variabilidad espacial y temporal de los índices biológicos (IBMWP, IASPT y EPT) en cuencas de pequeño tamaño (el 40 \% menores de $10 \mathrm{~km}^{2}$ ), basado en los datos de 26 estaciones de muestreo durante un periodo de 13 años. Se ha determinado cuales son las principales características que determinan la variabilidad de las estaciones de muestreo (PCA). Posteriormente se ha evaluado la respuesta de los índices biológicos respecto a las características de las subcuencas y la intensidad de los impactos (RDA). Los principales resultados indican que los índices biológicos están influenciados por la altitud, la temporalidad, la existencia de vegetación de fondo de valle y la ganadería, además de otros factores e impactos menos relevantes. Además, la altitud resulta un factor limitante en cuanto a la diversidad de macroinvertebrados fluviales en las estaciones más elevadas. De este modo, los índices bióticos responden tanto a las características de la subcuenca como a los posibles impactos presentes en ellas. No obstante, los límites entre clases de calidad del IBMWP establecidos por la legislación para el tipo de ríos de montaña mediterránea silícea (R-T11) son poco sensibles en lo que se refiere a áreas de montaña relativamente bien conservadas, por lo que se proponen unos umbrales más restrictivos y mejor adaptados para poder detectar los impactos en las subcuencas fluviales del Parque Nacional de la Sierra de Guadarrama.

Palabras clave: índices biológicos, macroinvertebrados acuáticos, espacios protegidos, estado ecológico, Sierra de Guadarrama, seguimiento, IBMWP

\section{ABSTRACT}

River biological indices in protected areas: are the all-purpose quality classes adequate?

The National Parks Master Plan specifies that the monitoring of ecological status must be carried out according to the parameters of the Water Framework Directive (WFD). However, mountain National Parks are characterized by small basins $(<10$ $\mathrm{km}^{2}$ ) that are outside the scope of the WFD. The objective of this article is to assess whether the biotic indices usually used by the WFD are appropriate to assess the ecological status of small and generally well conserved basins in protected mountain areas. For this purpose, the results of the monitoring of ecological status of the main rivers in the Sierra de Guadarrama National Park are presented. The spatial and temporal variability of the biological indices (IBMWP, IASPT and EPT) in small basins (generally less than $10 \mathrm{~km}^{2}$ ) has been evaluated, based on data from 26 sampling stations over a period of 13 years. The main characteristics that determine the variability of the sampling stations have been established (PCA). Afterwards, the response of the biological indices with respect to the characteristics of the sub-basins and the intensity of the impacts was evaluated (RDA). The main results indicate that the biological indices are influenced by altitude, seasonality, the existence of 
lowland vegetation and livestock, in addition to other less relevant factors and impacts. In addition, altitude is a limiting factor for the diversity of fluvial macroinvertebrates at the most elevated stations. In this way, the biotic indices respond both to the characteristics of the subbasin and to the possible impacts present in them. Nevertheless, the boundaries between IBMWP quality classes established by legislation for the type of Mediterranean silica mountain rivers ( $R$-T11) are not very sensitive with regard to relatively well conserved mountain areas, so more restrictive and better adapted thresholds are proposed in order to detect impacts in the river sub-basins of the Sierra de Guadarrama National Park.

Key words: biological indices, aquatic macroinvertebrates, protected areas, Sierra de Guadarrama, ecological status, monitoring, IBMWP

\section{INTRODUCCIÓN}

En los últimos años la aplicación de la Directiva Marco del Agua (DMA) ha permitido estandarizar una serie de metodologías para la caracterización y seguimiento del estado ecológico en ecosistemas fluviales (Prat \& Munné, 2014). Estas metodologías descansan en gran medida en el cálculo de una serie de índices que se calculan a partir de diferentes comunidades biológicas. En el caso de los ecosistemas fluviales, las comunidades biológicas de macroinvertebrados fluviales son las más ampliamente utilizadas en la evaluación del estado ecológico (Alba-Tercedor et al., 2002; MAGRAMA, 2013, 2015; Prat \& Munné, 2014). En España, el índice IBMWP (Alba-Tercedor et al., 2002) es uno de los más utilizados y ha demostrado ser adecuado para determinar el estado ecológico de los ríos (Munné \& Prat, 2009; Couto-Mendoza et al., 2015). En este sentido, las Confederaciones Hidrográficas han diseñado amplias redes de control y seguimiento, en función de las condiciones de referencia establecidas en la planificación hidrológica para cada ecotipo fluvial (Pujante et al., 2016).

Sin embargo, la aplicación a una escala espacio-temporal tan amplia ha obviado los pequeños cursos fluviales de menor entidad. Este tipo de cuencas es precisamente las que aparecen habitualmente en los espacios protegidos de montaña, en los que el uso de los macroinvertebrados acuáticos como indicadores puede ser de gran utilidad para la gestión y conservación. Así, se han empleado los macroinvertebrados para evaluar el estado ecológico de sus ríos tanto en el Parque Nacional de Ordesa y Monte Perdido (Valladolid et al., 2015) como en el Parque Nacional de Picos de Europa (Barquín et al., 2015). En el de Sierra
Nevada se ha estudiado el grupo de los tricópteros en relación al cambio climático (Zamora-Muñoz et al., 2011; Sáinz Bariáin, 2014).

Lo que no está tan claro es que los límites desarrollados para la evaluación de toda la red hidrográfica española sean adecuados para evaluar este tipo de cuencas de pequeño tamaño situadas en espacios protegidos de montaña. Ya se ha señalado (Valladolid et al., 2010) la necesidad de realizar estudios con mayor detalle sobre calidad de agua a nivel de pequeñas cuencas, frente a los estudios que abarcan el nivel de gran cuenca hidrográfica. Por otro lado, existen trabajos puntuales en cuencas pequeñas cuestionando la idoneidad de los índices establecidos (Ruiz-García \& Ferreras-Romero, 2015), o se han propuesto unas estaciones de referencia específicas para un ámbito concreto (Bordallo López \& Casado Sancho, 2012).

La Sierra de Guadarrama se caracteriza por albergar cientos de kilómetros de cursos fluviales, mayoritariamente arroyos de escasa entidad (generalmente órdenes 1 y 2). De los 32 ecotipos caracterizados oficialmente, se han cartografiado dos en la Sierra de Guadarrama: ríos de montaña mediterránea silícea (R- T11), que son los predominantes (Toro et al., 2009a), y ríos de alta montaña (R-T27) en su vertiente norte (Toro et al., 2009b). En el ámbito de la Comunidad de Madrid los únicos cursos fluviales que pudieran ser incluidos como estaciones de referencia por su estado de conservación se encuentran en la Sierra de Guadarrama (Bordallo López \& Casado Sancho, 2012). Sin embargo, también algunos de ellos están sometidos a presiones por el uso público, debido principalmente a la proximidad de la gran extensión urbana de Madrid, así como el efecto llamada del turismo hacia los espacios 
protegidos. Además, la ganadería extensiva que se practica en la Sierra de Guadarrama en ocasiones genera alteraciones de la vegetación de ribera y produce contaminación orgánica difusa. Los aprovechamientos forestales en ciertos ámbitos favorecen las especies madereras frente al bosque de ribera (Rubio-Romero \& Granados, 2013). No obstante, en términos generales los espacios protegidos de la Sierra de Guadarrama han contribuido a la conservación de los ecosistemas fluviales (Casado et al., 2011).

El objetivo de este artículo es 1) evaluar la variabilidad espacial y temporal de los índices biológicos en cuencas de pequeño tamaño situadas en alta montaña mediterránea; 2) valorar si los índices biológicos de uso general tienen la suficiente sensibilidad para detectar impactos más o menos difusos en este tipo de cuencas; 3 ) determinar si la clasificación del estado ecológico establecida para el ecotipo R-T11 es de utilidad en el sistema de seguimiento del Parque Nacional de la Sierra de Guadarrama; y 4) proponer unos umbrales específicos para la determinación del estado ecológico de los cursos fluviales de este espacio protegido.

\section{MATERIAL Y MÉTODOS}

\section{Área de estudio}

La Sierra de Guadarrama constituye una de las alineaciones montañosas del Sistema Central Español. La vegetación predominante de la Sierra, de acuerdo con un gradiente altitudinal, está constituida por pastos de montaña y matorrales de altura (piornales y enebrales), así como

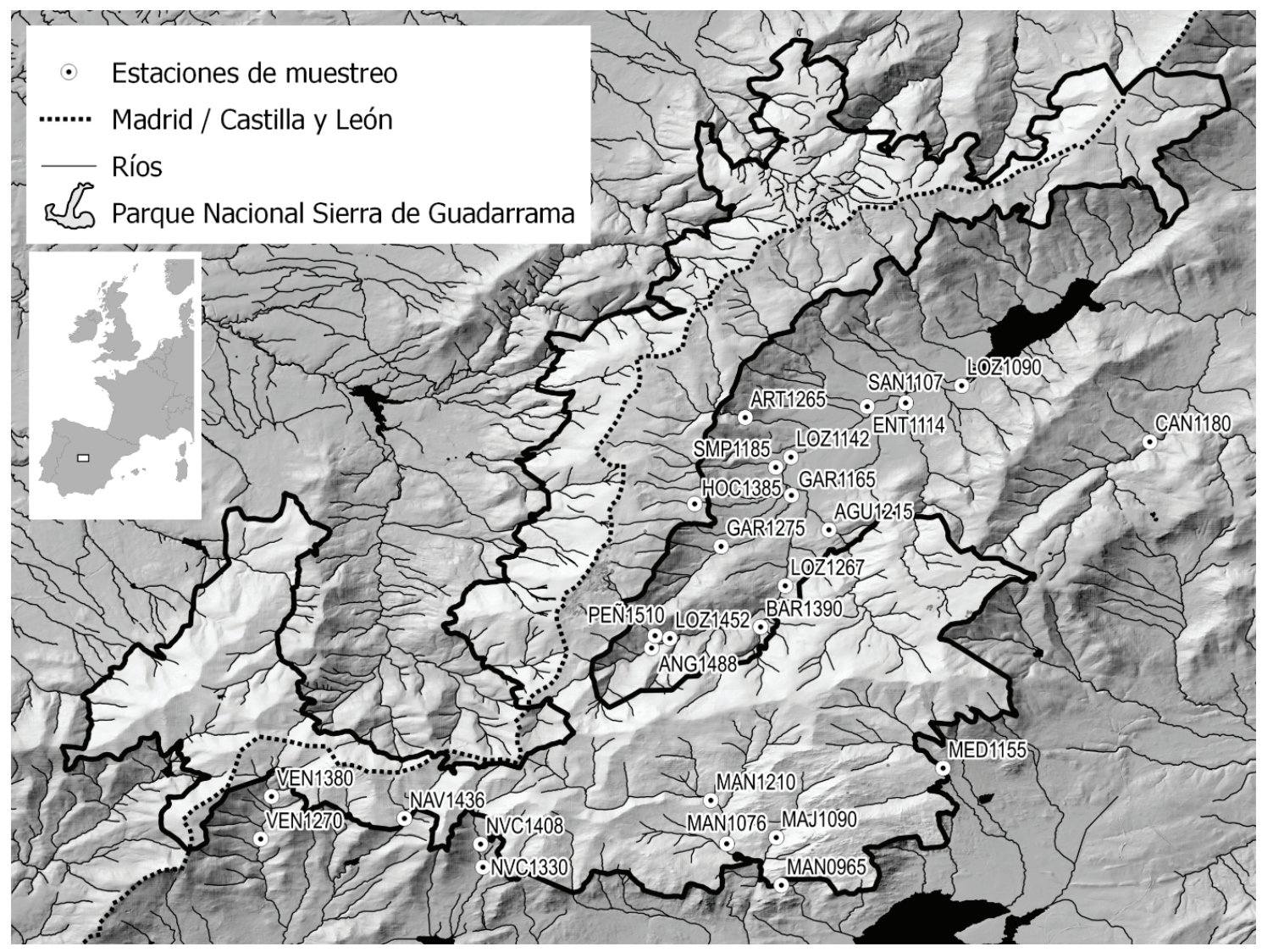

Figura 1. Localización del Parque Nacional de la Sierra de Guadarrama y estaciones de muestreo de la red de seguimiento (Comunidad de Madrid). Localization of the Sierra de Guadarrama National Park and sampling stations of the monitoring network (Madrid Region). 
pinares de Pinus sylvestris (1200-2000 m s.n.m.) y, en menor proporción, melojares de Quercus pyrenaica (1000-1200 m s.n.m.) y otras formaciones arbóreas. Son frecuentes, además, los afloramientos rocosos. El clima del Parque Nacional de la Sierra de Guadarrama se puede asociar a un clima mediterráneo muy frío $\mathrm{y}$ verano templado, con una temperatura media del mes más frío de $-0.7{ }^{\circ} \mathrm{C}$ y de $16.4{ }^{\circ} \mathrm{C}$ del mes más cálido, en enero y julio respectivamente (Observatorio AEMET Puerto de Navacerrada, 1890 m s.n.m.). La precipitación media anual es de 1325 $\mathrm{mm}$, con una acusada mediterraneidad. La geología predominante está constituida por un sustrato hercínico ácido, dominado por ortogneis glandular, granitos y granodioritas, apareciendo en menor proporción en los fondos de valle depósitos aluviales cuaternarios y rocas sedimentarias (margas, calizas y dolomías).

Cerca de $340 \mathrm{~km}^{2}$ se declararon parque nacional en 2013, el $64 \%$ en la Comunidad de Madrid y el $36 \%$ en la Comunidad de Castilla y León. El parque nacional incluye $337 \mathrm{~km}$ de cursos de agua, agrupados en las cuencas de los ríos Lozoya, Manzanares y Guadarrama (Comunidad de Madrid); y Eresma, Pirón, Moros y Cega (Castilla y León). Los ríos son de cabecera de alta montaña, caracterizados por un régimen pluvionival. El seguimiento de los ecosistemas fluviales en la parte madrileña de la Sierra de Guadarrama se inició en 2005, con el objetivo de evaluar el estado ecológico de los arroyos del Parque Natural de Peñalara y su Zona Periférica de Protección (Rubio-Romero \& Granados, 2013). Para ello se seleccionaron 15 estaciones de muestreo (tramos de $100 \mathrm{~m}$ ) en el Alto Lozoya. En 2014, tras la inclusión del Parque Natural en el Parque Nacional de la Sierra de Guadarrama (en adelante PNSG), se amplió el seguimiento a otros 11 puntos de la Sierra de Guadarrama (fundamentalmente cuencas del Manzanares y Guadarrama en la Comunidad de Madrid, Fig. 1 y Tabla 1A). De las 26 estaciones, $10(38.5 \%)$ drenan superficies menores de $10 \mathrm{~km}^{2}$. La actual red de seguimiento está destinada a identificar posibles impactos en el parque nacional y proporcionar un indicador del estado de conservación de los principales ríos y arroyos. El código de cada estación de muestreo está formado por una cadena alfanumérica de 3 letras (topónimo del curso fluvial) y 4 números (altitud media del tramo muestreado).

\section{Índices biológicos fluviales}

Se han realizado dos campañas de muestreo anuales, en primavera (entre el 30 de marzo y el 30 de junio), y en verano (entre el 15 de julio y el 18 de septiembre). En cada estación de muestreo se calcularon diversos índices relativos a la composición de la comunidad de macroinvertebrados fluviales (IBMWP, IASPT, EPT; Alba-Tercedor et al., 2002, 2005; AQEM Consortium, 2002; Jáimez-Cuéllar et al., 2002; MAGRAMA, 2013; en adelante índices biológicos cuando se traten conjuntamente). Para la recolección de macroinvertebrados, se empleó una red de mano de $30 \mathrm{x}$ $25 \mathrm{~cm}$ tipo kicker con tamaño de luz de malla de $350 \mu \mathrm{m}$. El esfuerzo de muestreo fue de 20 kicks, distribuidos proporcionalmente a la abundancia de los tipos de hábitat presentes. La muestra se fijó con alcohol al $70 \%$ para su posterior determinación en laboratorio.

Las condiciones de referencia se han obtenido del Real Decreto 817/2015 por el que se establecen los criterios de seguimiento y evaluación del estado de las aguas superficiales (MAGRAMA, 2015; Pujante et al., 2016). En el caso de la parte madrileña de la Sierra de Guadarrama se corresponde con el ecotipo R-T11, ríos de montaña mediterránea silícea, cuyo valor de referencia para el IBMWP es de 193 puntos, con los límites de cambio de clase en RCE 0.82 (muy bueno/bueno) y 0.50 (bueno/moderado). Los datos de índices biológicos correspondientes al resto de la cuenca del Tajo para el período 2006-2014 se han obtenido de página web de la confederación hidrográfica (Confederación Hidrográfica del Tajo, 2018).

\section{Caracterización de las cuencas e impactos}

Las características de las cuencas de drenaje de cada estación de muestreo (Tabla 1A) se han obtenido del modelo digital del terreno a escala 1:5000 del PNOA (Comunidad de Madrid, 2018). La vegetación presente en cada cuenca (Tabla 1B) se ha obtenido mediante la reclasificación (simplificación) de la cartografía de vege- 
Tabla 1. A) Estaciones de muestreo. Nombre del curso de agua y cuenca a la que pertenece. Principales características hidromorfológicas de la cuenca. Para los análisis estadísticos, la orientación se ha descompuesto en dos variables: northness $=\cos ($ orientación), eastness $=\sin ($ orientación). *En 2015 en la mayoría de estaciones sólo se realizó la campaña de verano. B) Porcentaje de cada tipo de vegetación en la cuenca y estimación del nivel de impactos. A) Sampling stations. Name of the watercourse and basin to which it belongs. Main hydromorphological characteristics of the basin. For statistical analyses, the orientation has been decomposed into two variables: northness $=\cos$ (orientation), eastness $=\sin \left(\right.$ orientation). ${ }^{*}$ In 2015 , only the summer campaign was carried out in most of the stations. B) Percentage of each type of vegetation in the basin and estimation of the impact level.

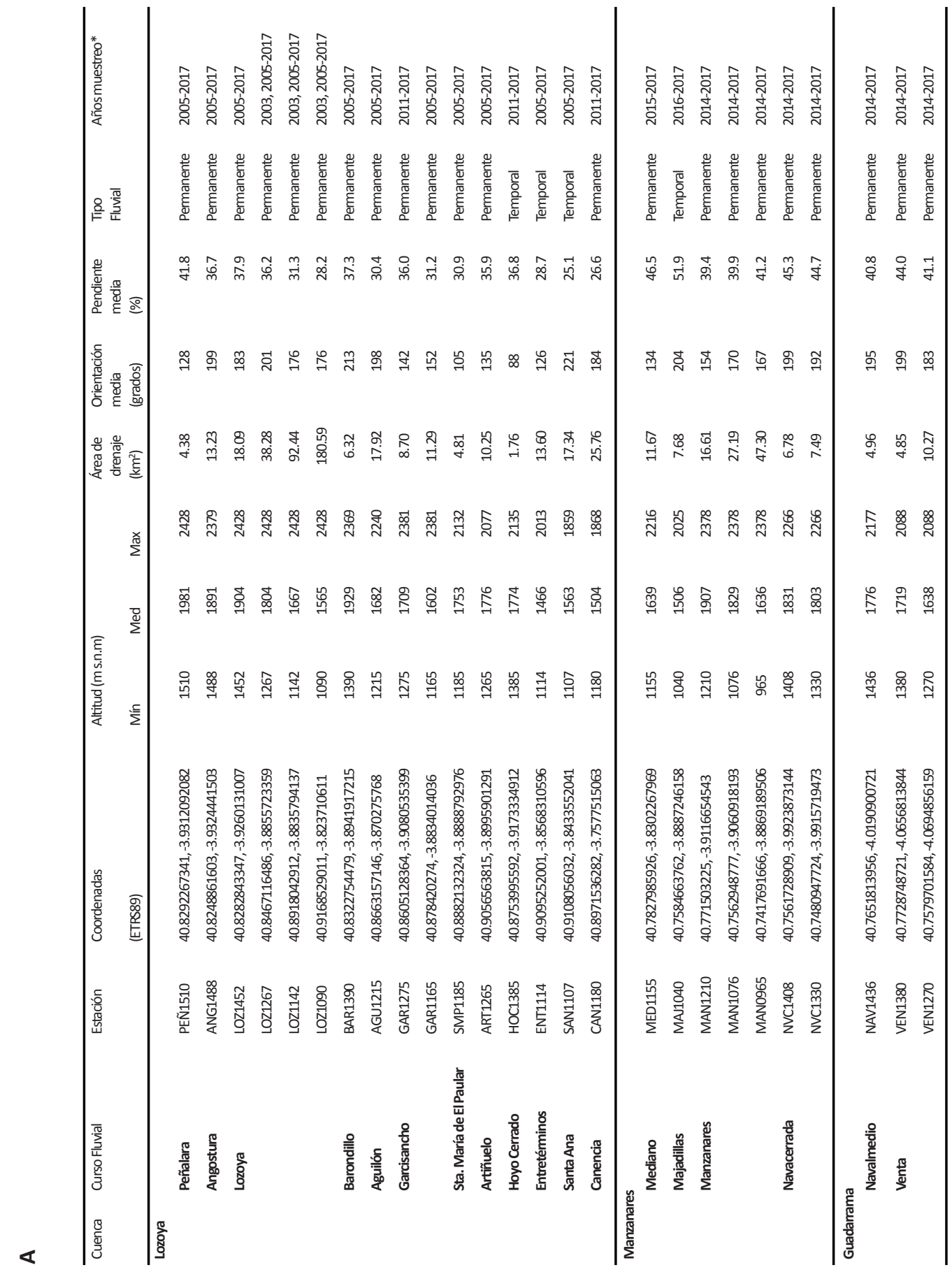




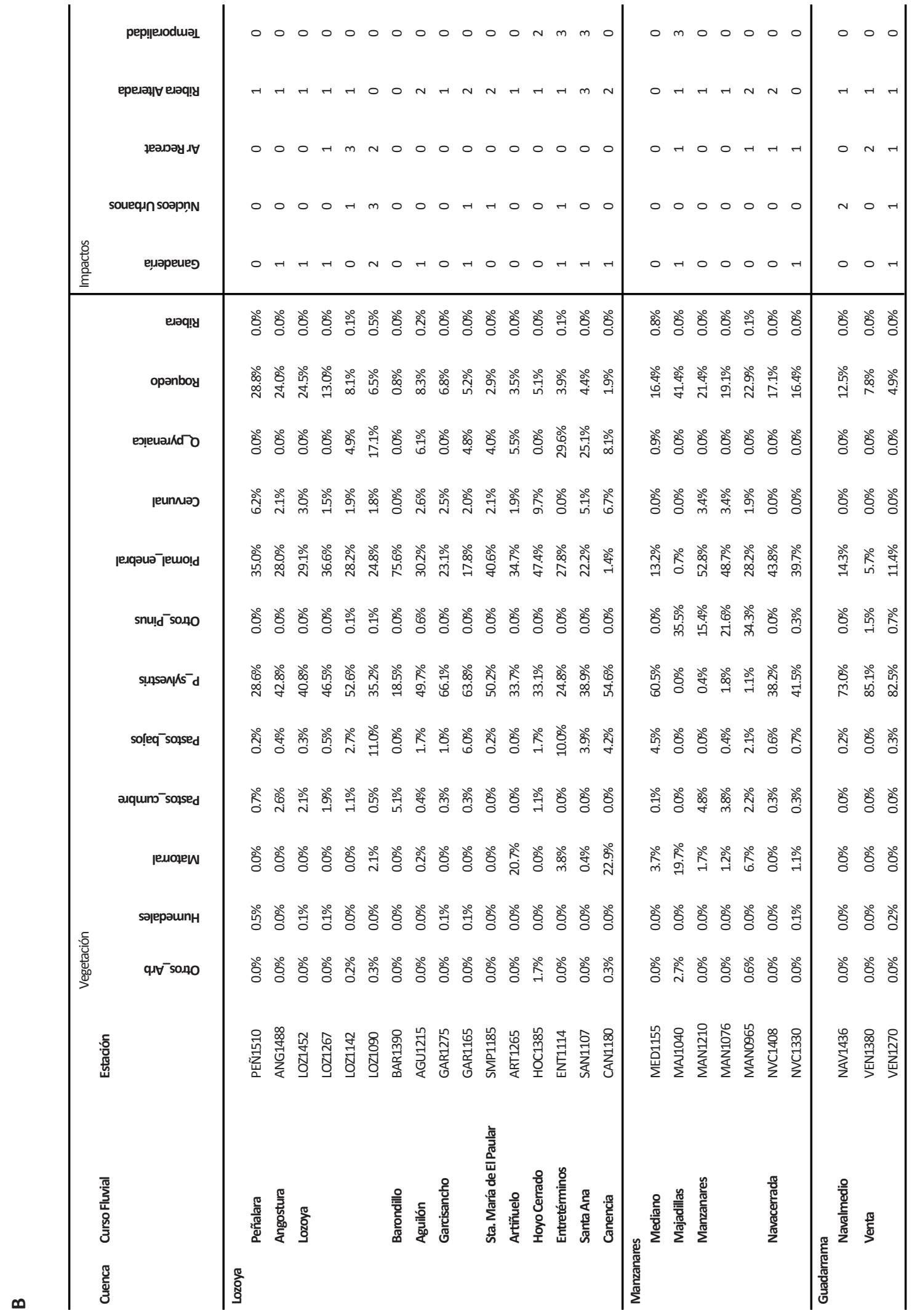


tación 1:25 000 de la Sierra de Guadarrama (Muñoz Municio et al., 2004).

Se ha estimado, a nivel de subcuenca, el grado del impacto causado por la ganadería, la existencia de áreas recreativas (o gran actividad de baño), la presencia de núcleos urbanizados y la degradación del bosque de ribera (muchas veces originada por la explotación forestal tradicional). A cada impacto se le ha asignado un valor de 0 a 3 , dependiendo de la intensidad de éste según el criterio consensuado del personal técnico del parque nacional (Tabla 1B). Además, en los análisis estadísticos se ha incluido la temporalidad como un factor de estrés adicional, aunque se trata de una característica natural. El grado de impacto total se ha considerado como la suma de todos ellos. Otros impactos conocidos en la Sierra de Guadarrama, cómo la presencia de carreteras en la cuenca, pequeños represamientos o detracciones de agua para riego, no se han considerado en el análisis estadístico al ser mucho menos relevantes para el estado ecológico de los ríos serranos, aunque sí se han tenido en cuenta para la selección de las estaciones más naturalizadas.

\section{Análisis estadísticos}

El tratamiento estadístico de los datos se realizó con el software R (R Core Team, 2017). Para evaluar las diferencias entre las medias de los índices bióticos se empleó un test no paramétrico (Wilcoxon de dos muestras no pareadas; paquete R-Commander; Fox, 2005). Tanto la respuesta de los índices biológicos a la altitud como la correlación entre los índices biológicos se determinó mediante un modelo lineal mixto (LMM) con el paquete lme4 (Bates et al., 2015), controlando la variabilidad en las estaciones de muestreo y el año de muestreo (efectos aleatorios). Para la realización del modelo se comprobó la normalidad y homocedasticidad de los datos (Zuur et al., 2010). El coeficiente de correlación R2 (marginal y condicional) de los LMM se determinó de acuerdo a Nakagawa \& Schielzeth (2012) con el paquete MuMIn (Bartoń, 2018). Las tendencias interanuales de los índices biológicos se han estimado con MAKESENS (Salmi et al., 2002). Se realizó un análisis de componentes principales (PCA) mediante el paquete FactoMineR (Lê et al., 2008) sobre los datos normalizados de las características morfométricas de la cuenca y el tipo de vegetación, con el objetivo de determinar qué factores explican la variabilidad de las estaciones de muestreo. Posteriormente, con el paquete vegan (Oksanen et al., 2018) se realizó un análisis de redundancia (RDA) para explorar las relaciones entre una matriz de datos con los índices biológicos (respuesta) y otra matriz de datos con las características de la cuenca y los impactos estimados en cada una de ellas (explicativa). Para evitar la sobredeterminación del modelo debido a un exceso de variables explicativas, se utilizó en el RDA sólo una selección de variables. Esta selección incluye 5 variables relacionadas con impactos (Tabla 1B), así como tres variables relacionadas con las características de cuenca. Para elegir estas tres últimas se han considerado sólo las variables de cuenca cuya suma del coseno cuadrado $\left(\operatorname{Cos}^{2}\right)$ de los dos primeros ejes del PCA fuese superior a 0.6 , y cuyas correlaciones lineales entre las distintas variables tuviese un $\mathrm{R}^{2}<0.5$. La suma de $\operatorname{Cos}^{2}$ permite evitar errores de interpretación debidos a un efecto de la proyección, por lo que es un buen criterio para seleccionar las variables más relevantes (Abdi \& Williams, 2010). De esta manera, se evalúa si los impactos de cuenca afectan a las puntuaciones de los índices biológicos en el contexto de variabilidad de cuencas de la Sierra de Guadarrama. La significación global del modelo del RDA y la significación de las variables respuesta se ha comprobado con un test de permutación de Monte Carlo con 999 iteraciones.

\section{RESULTADOS}

\section{Índices biológicos}

\section{Alto Lozoya}

Entre 2005 y 2017 se han calculado 140 índices IBMWP durante las campañas de primavera de los ríos permanentes del Alto Lozoya (Tabla 2), obteniendo una media de 174 puntos (RCE 0.90 ). El $80.7 \%$ está por encima de los 158 puntos (RCE 0.82) que marca el límite inferior del estado ecológico muy bueno, y el $17.1 \%$ obtiene una puntuación mayor que el estado de referencia (193). No se han obtenido valores correspondien- 
Tabla 2. Resultados del IBMWP, RCE, IASPT y EPT en primavera en el Alto Lozoya. Results of IBMWP, EQR, IASPT and EPT during spring in Alto Lozoya.

\begin{tabular}{|c|c|c|c|c|c|c|c|c|c|c|c|c|c|c|c|c|}
\hline Curso Fluvial & Estación & Índice & 유 & ర్ల & ఫ్రి & ర్లి & ఫ્તి & 옴 & ్ㅗㅁ & శ్ & 芩 & 总 & 岂 & 芩 & ิㅗㅁ & $\overline{\mathbb{8}}$ \\
\hline \multirow[t]{4}{*}{ Peñalara } & PEÑ1510 & IBMWP & 164 & 188 & 153 & 165 & 155 & 162 & 162 & 175 & 161 & 182 & & 179 & 192 & 169.8 \\
\hline & & RCE & 0.91 & 1.04 & 0.85 & 0.92 & 0.86 & 0.90 & 0.90 & 0.97 & 0.89 & 1.01 & & 0.99 & 1.07 & 0.9 \\
\hline & & IASPT & 6.3 & 7.5 & 6.7 & 6.9 & 7.0 & 6.5 & 6.2 & 6.7 & 6.2 & 6.7 & & 6.6 & 6.6 & 6.7 \\
\hline & & EPT & 22 & 24 & 18 & 20 & 15 & 18 & 15 & 18 & 18 & 22 & & 17 & 20 & 18.9 \\
\hline \multirow[t]{4}{*}{ Angostura } & ANG1488 & IBMWP & 206 & 181 & 181 & 192 & 183 & 185 & 189 & 197 & 182 & 213 & & 205 & 184 & 191.5 \\
\hline & & RCE & 1.14 & 1.01 & 1.01 & 1.07 & 1.02 & 1.03 & 1.05 & 1.09 & 1.01 & 1.18 & & 1.14 & 1.02 & 1.1 \\
\hline & & IASPT & 6.2 & 6.5 & 6.7 & 6.9 & 6.8 & 6.4 & 6.3 & 6.4 & 6.1 & 6.5 & & 6.4 & 6.8 & 6.5 \\
\hline & & EPT & 21 & 18 & 17 & 21 & 19 & 18 & 18 & 18 & 14 & 17 & & 18 & 16 & 17.9 \\
\hline \multirow[t]{16}{*}{ Lozoya } & LOZ1090 & IBMWP & 106 & 117 & 131 & 148 & 176 & 124 & 126 & 114 & 129 & 131 & & 109 & 129 & 128.3 \\
\hline & & RCE & 0.59 & 0.65 & 0.73 & 0.82 & 0.98 & 0.69 & 0.70 & 0.63 & 0.72 & 0.73 & & 0.61 & 0.72 & 0.7 \\
\hline & & IASPT & 5.0 & 6.2 & 6.6 & 5.9 & 6.3 & 6.5 & 6.3 & 5.7 & 5.6 & 6.2 & & 5.5 & 6.1 & 6.0 \\
\hline & & EPT & 12 & 13 & 13 & 15 & 15 & 12 & 13 & 11 & 10 & 13 & & 9 & 12 & 12.3 \\
\hline & LOZ1142 & IBMWP & 141 & 159 & 146 & 175 & 203 & 160 & 173 & 173 & 180 & 195 & & 180 & 165 & 170.8 \\
\hline & & RCE & 0.78 & 0.88 & 0.81 & 0.97 & 1.13 & 0.89 & 0.96 & 0.96 & 1.00 & 1.08 & & 1.00 & 0.92 & 0.9 \\
\hline & & IASPT & 5.9 & 6.9 & 6.3 & 7.0 & 6.3 & 5.9 & 6.2 & 6.2 & 6.7 & 6.7 & & 6.0 & 6.1 & 6.4 \\
\hline & & EPT & 15 & 17 & 17 & 17 & 16 & 16 & 15 & 16 & 18 & 21 & & 15 & 15 & 16.5 \\
\hline & LOZ1267 & IBMWP & 160 & 118 & 163 & 163 & 150 & 158 & 178 & 170 & 158 & 157 & & 172 & 185 & 161.0 \\
\hline & & RCE & 0.89 & 0.66 & 0.91 & 0.91 & 0.83 & 0.88 & 0.99 & 0.94 & 0.88 & 0.87 & & 0.96 & 1.03 & 0.9 \\
\hline & & IASPT & 6.2 & 6.2 & 6.5 & 6.5 & 6.0 & 6.6 & 5.9 & 6.1 & 5.9 & 6.5 & & 6.6 & 6.4 & 6.3 \\
\hline & & EPT & 21 & 16 & 18 & 18 & 17 & 19 & 18 & 18 & 17 & 15 & & 17 & 17 & 17.6 \\
\hline & LOZ1452 & IBMWP & 191 & 180 & & 188 & 178 & 165 & 162 & 192 & 185 & 189 & & 189 & 197 & 183.3 \\
\hline & & RCE & 1.06 & 1.00 & & 1.04 & 0.99 & 0.92 & 0.90 & 1.07 & 1.03 & 1.05 & & 1.05 & 1.09 & 1.0 \\
\hline & & IASPT & 6.6 & 6.7 & & 7.0 & 6.4 & 6.3 & 5.8 & 6.6 & 6.6 & 7.0 & & 6.3 & 6.4 & 6.5 \\
\hline & & EPT & 22 & 21 & & 18 & 17 & 19 & 14 & 19 & 16 & 19 & & 18 & 18 & 18.3 \\
\hline \multirow[t]{4}{*}{ Barondillo } & BAR1390 & IBMWP & 197 & 170 & 186 & 169 & 182 & 177 & 168 & 175 & 175 & 146 & & 163 & 162 & 172.5 \\
\hline & & RCE & 1.09 & 0.94 & 1.03 & 0.94 & 1.01 & 0.98 & 0.93 & 0.97 & 0.97 & 0.81 & & 0.91 & 0.90 & 1.0 \\
\hline & & IASPT & 6.6 & 6.5 & 7.2 & 7.0 & 6.7 & 6.3 & 6.7 & 6.3 & 6.3 & 6.1 & & 6.5 & 6.0 & 6.5 \\
\hline & & EPT & 23 & 21 & 21 & 20 & 16 & 19 & 18 & 16 & 17 & 16 & & 16 & 17 & 18.3 \\
\hline \multirow[t]{4}{*}{ Aguilón } & AGU1215 & IBMWP & 207 & 178 & 182 & 243 & 209 & 214 & 211 & 218 & 227 & 220 & & 193 & 197 & 208.3 \\
\hline & & RCE & 1.15 & 0.99 & 1.01 & 1.35 & 1.16 & 1.19 & 1.17 & 1.21 & 1.26 & 1.22 & & 1.07 & 1.09 & 1.2 \\
\hline & & IASPT & 6.3 & 6.4 & 6.5 & 6.6 & 6.1 & 6.3 & 6.4 & 6.2 & 6.3 & 6.3 & & 6.7 & 6.4 & 6.4 \\
\hline & & EPT & 22 & 19 & 20 & 24 & 19 & 18 & 20 & 21 & 20 & 19 & & 18 & 16 & 19.7 \\
\hline \multirow[t]{8}{*}{ Garcisancho } & GAR1165 & IBMWP & 142 & 215 & 160 & 183 & 183 & 174 & 179 & 201 & 199 & 156 & & 191 & 168 & 179.3 \\
\hline & & RCE & 0.79 & 1.19 & 0.89 & 1.02 & 1.02 & 0.97 & 0.99 & 1.12 & 1.11 & 0.87 & & 1.06 & 0.93 & 1.0 \\
\hline & & IASPT & 5.7 & 6.7 & 5.9 & 6.3 & 6.5 & 6.4 & 6.4 & 6.1 & 6.4 & 6.2 & & 6.8 & 6.5 & 6.3 \\
\hline & & EPT & 14 & 19 & 14 & 16 & 19 & 17 & 16 & 18 & 19 & 16 & & 21 & 17 & 17.2 \\
\hline & GAR1275 & IBMWP & & & & & & & 180 & 209 & 178 & 177 & & 163 & 173 & 180.0 \\
\hline & & RCE & & & & & & & 1.00 & 1.16 & 0.99 & 0.98 & & 0.91 & 0.96 & 1.0 \\
\hline & & IASPT & & & & & & & 6.4 & 6.3 & 6.6 & 6.8 & & 6.8 & 6.4 & 6.6 \\
\hline & & EPT & & & & & & & 16 & 18 & 15 & 21 & & 17 & 16 & 17.2 \\
\hline \multirow[t]{4}{*}{ Sta. M. Paular } & SMP1185 & IBMWP & 172 & 200 & 176 & 168 & 165 & 183 & 154 & 186 & 145 & 189 & & 158 & 189 & 173.8 \\
\hline & & RCE & 0.96 & 1.11 & 0.98 & 0.93 & 0.92 & 1.02 & 0.86 & 1.03 & 0.81 & 1.05 & & 0.88 & 1.05 & 1.0 \\
\hline & & IASPT & 6.1 & 6.9 & 6.8 & 6.7 & 6.9 & 7.0 & 5.9 & 6.4 & 6.3 & 6.8 & & 6.9 & 6.5 & 6.6 \\
\hline & & EPT & 19 & 21 & 19 & 16 & 17 & 18 & 16 & 18 & 13 & 21 & & 17 & 20 & 17.9 \\
\hline \multirow[t]{4}{*}{ Artiñuelo } & ART1265 & IBMWP & 179 & 172 & 177 & 166 & 164 & 170 & 182 & 177 & 200 & 145 & & 162 & 181 & 172.9 \\
\hline & & RCE & 0.99 & 0.96 & 0.98 & 0.92 & 0.91 & 0.94 & 1.01 & 0.98 & 1.11 & 0.81 & & 0.90 & 1.01 & 1.0 \\
\hline & & IASPT & 6.2 & 6.1 & 6.3 & 6.4 & 6.3 & 6.3 & 6.5 & 6.1 & 6.9 & 6.3 & & 6.8 & 6.5 & 6.4 \\
\hline & & EPT & 19 & 19 & 18 & 19 & 18 & 17 & 18 & 20 & 20 & 16 & & 15 & 19 & 18.2 \\
\hline \multirow[t]{4}{*}{ Hoyo Cerrado } & HOC1385 & IBMWP & & & & & & & 158 & 156 & 156 & 170 & 133 & 165 & 158 & 156.6 \\
\hline & & RCE & & & & & & & 0.88 & 0.87 & 0.87 & 0.94 & 0.74 & 0.92 & 0.88 & 0.9 \\
\hline & & IASPT & & & & & & & 6.1 & 6.0 & 6.8 & 6.5 & 6.3 & 6.3 & 6.3 & 6.3 \\
\hline & & EPT & & & & & & & 15 & 14 & 16 & 16 & 13 & 16 & 17 & 15.3 \\
\hline \multirow[t]{4}{*}{ Entretérminos } & ENT1114 & IBMWP & & 89 & 89 & 97 & 84 & 85 & 96 & 92 & 77 & 96 & 119 & 101 & 104 & 94.1 \\
\hline & & RCE & & 0.49 & 0.49 & 0.54 & 0.47 & 0.47 & 0.53 & 0.51 & 0.43 & 0.53 & 0.66 & 0.56 & 0.58 & 0.5 \\
\hline & & IASPT & & 6.8 & 6.8 & 6.1 & 6.0 & 6.5 & 6.0 & 5.8 & 5.1 & 6.9 & 6.3 & 6.3 & 5.5 & 6.2 \\
\hline & & EPT & & 10 & 10 & 11 & 12 & 11 & 11 & 11 & 7 & 12 & 12 & 11 & 9 & 10.6 \\
\hline Santa Ana & SAN1107 & IBMWP & & 94 & 87 & 104 & & 59 & 92 & 65 & 81 & 73 & & 90 & & 82.8 \\
\hline & & RCE & & 0.52 & 0.48 & 0.58 & & 0.33 & 0.51 & 0.36 & 0.45 & 0.41 & & 0.50 & & 0.5 \\
\hline & & IASPT & & 5.9 & 5.8 & 6.1 & & 5.9 & 6.1 & 5.0 & 5.8 & 5.6 & & 5.3 & & 5.7 \\
\hline & & EPT & & 9 & 9 & 13 & & 6 & 10 & 7 & 9 & 8 & & 7 & & 8.7 \\
\hline
\end{tabular}


tes al estado ecológico moderado $(<97)$ en estaciones de ríos permanentes. En 18 de los 27 $(66.7 \%)$ casos con una valoración ecológica buena se trata de alguna estación en el eje del río Lozoya, particularmente (40.7 \%) en la estación a menor altitud (LOZ1090). Esta estación y otras dos situadas aguas arriba (LOZ1267 y PEÑ1510) son las únicas en las que nunca se ha superado el umbral de referencia. Por el contrario, la estación del Aguilón (AGU1215) supera el umbral de referencia en el $75 \%$ de los muestreos (media 208, máximo 243 puntos). Ninguna otra estación presenta un RCE medio > 1 en 12 años, aunque La Angostura (ANG1488) con 0.99 y en el Lozoya (LOZ1452) con 0.95 se aproximan considerablemente. De manera global, no se ha observado una tendencia significativa en la evolución del IBMWP en el Alto Lozoya. Sólo hay una estación (BAR1390) que muestra claramente un descenso (MAKESENS, $\mathrm{n}=12,-2.4 \pm 1.2$ puntos/año, $p<0.01$ ) en el IBMWP. Si se excluye
BAR1390 de las medias anuales, hay una cierta tendencia global a mejorar el IBMWP en el Alto Lozoya (MAKESENS, $\mathrm{n}=12,0.9 \pm 0.8$ puntos/año, $p<0.1)$. Tomando las cinco estaciones situadas en el eje principal del río Lozoya, se observa que el índice generalmente presenta valores más elevados conforme aumenta la altitud de la estación (Fig. 2A). No obstante, este patrón no se cumple si se tienen en cuenta todas las estaciones permanentes del Alto Lozoya (Fig. 2B). Por ejemplo, la tercera estación con menor valoración media en el Alto Lozoya es la situada a mayor altura (PEÑ1510).

Respecto a los ríos temporales, presentan claramente una menor puntuación del índice IBMWP en comparación con los ríos permanentes. En los temporales se reduce significativamente (Wilcoxon, $p<0.0001$ ) la valoración media hasta 106 puntos (RCE 0.55). Los dos cursos de agua que se secan completamente, el Entretérminos (ENT1114, 94 puntos) y el Santa

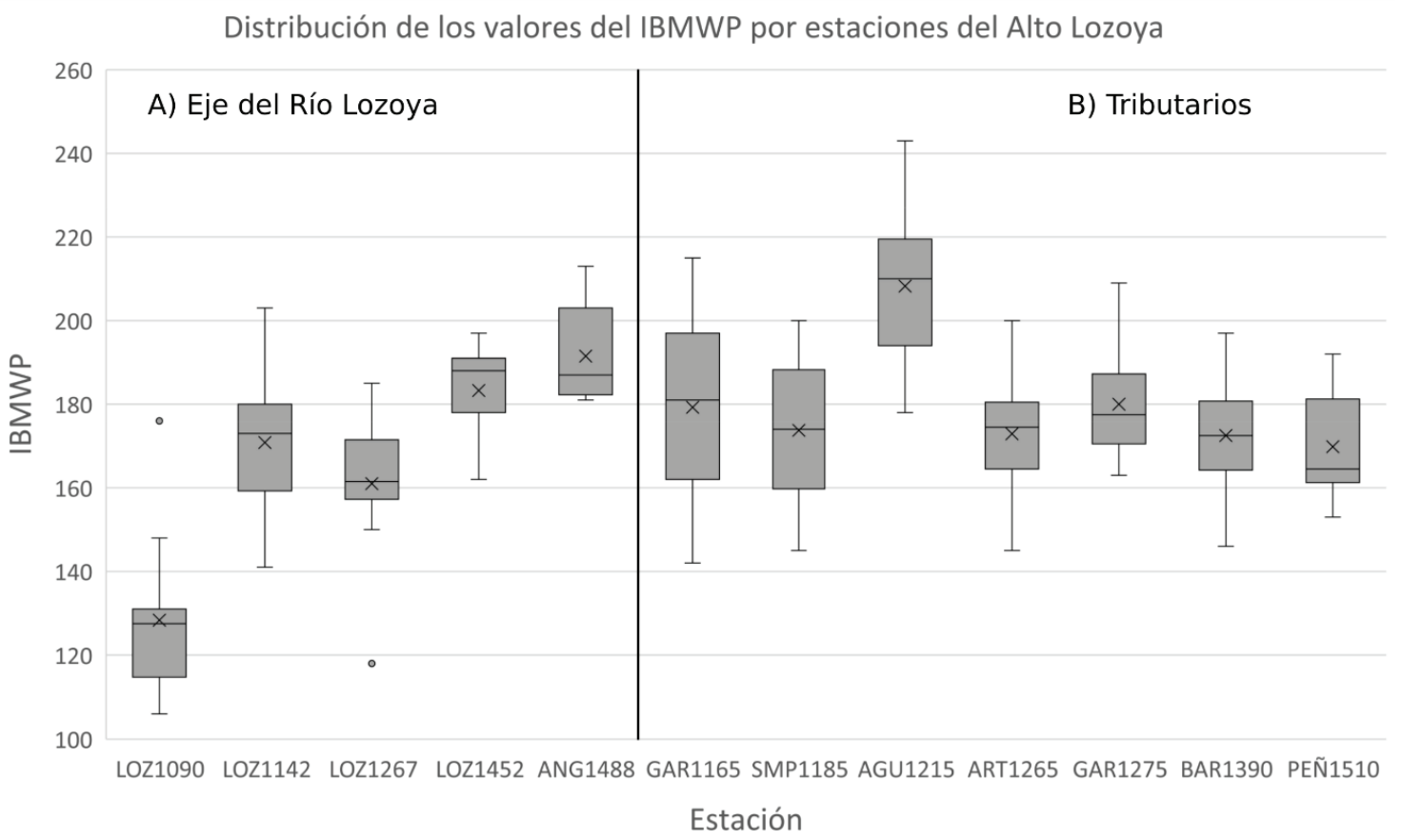

Figura 2. Distribución de los valores del IBMWP por estación de muestreo en los cursos fluviales permanentes: media, mediana, cuartiles, máximo, mínimo y puntos anómalos. A) estaciones del eje del río Lozoya. B) estaciones situadas en tributarios. Ordenadas de izquierda a derecha por altitud en ambos casos. Distribution of the IBMWP values by sampling station in the permanent streams: mean, median, quartiles, maximum, minimum and anomalous points. A) stations on the axis of the Lozoya River. B) stations located in tributaries. Ordered from left to right by altitude in both cases. 

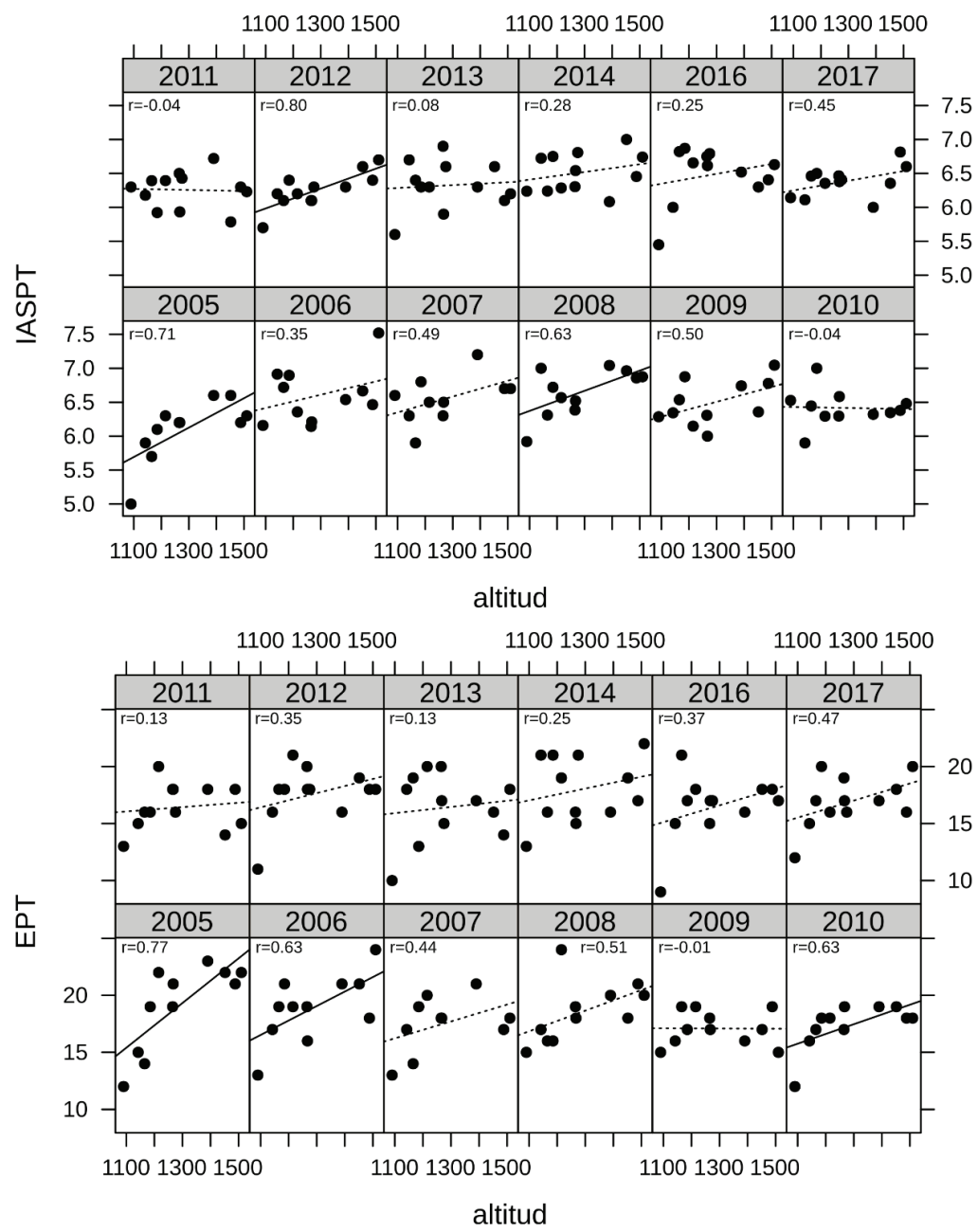

Figura 3. Correlación de IASTP (A) y el EPT (B) con la altitud. Se indica el valor de correlación para cada año. La línea no punteada indica correlaciones significativas $(p<0.05)$. Correlation of IASTP $(A)$ and EPT $(B)$ against altitude. It is shown the correlation value for each year. The non-dotted line means significant correlations $(\mathrm{p}<0.05)$.

Ana (SAN1107, 83 puntos), obtienen la peor puntuación media. El otro arroyo intermitente, Hoyocerrado, al presentar pozas con agua consigue mantener una puntuación media relativamente alta (HOC1385, 157 puntos).

En cuanto a la estacionalidad del índice, sólo es posible comparar aquellas estaciones que no se secan en verano. Así, en la campaña estival se produce un descenso significativo (Wilcoxon, $p<$ 0.001 ) de la media hasta 164 puntos (RCE 0.84) en los ríos permanentes. Las fechas en las que se obtiene una mayor puntuación del IBMWP y una menor variabilidad interanual están en torno a los primeros 20 días de junio.

Para las estaciones permanentes y la campaña de primavera, la media del IASPT fue de 6.42 en el Alto Lozoya (Tabla 2). Nuevamente, la estación que presenta una peor valoración media (5.99) es la de menor altitud del Alto Lozoya (LOZ1090). De hecho, todas las estaciones situadas por encima de 1270 m s.n.m. (junto con SMP1185) presentan un valor medio del IASPT superior a la media. Existe una correlación significativa entre el IASPT y la altitud (LMM, Fig. 3, 
$\left.\mathrm{n}=137, \mathrm{R}_{\mathrm{m}}{ }^{2}=0.11, \mathrm{R}_{\mathrm{c}}{ }^{2}=0.32, p<0.001\right) . \mathrm{El}$ IBMWP no presenta esta correlación significativa. El IASPT también presenta un descenso estacional significativo similar al del IBMWP, situándose en 6.03 como valor medio en verano (Wilcoxon, $p<0.0001$ ). Por otra parte, en los arroyos temporales el IASTP medio de primavera se reduce significativamente hasta 6.07 (Wilcoxon, $p<0.001)$.

El EPT (Tabla 2) presenta una media de 17.5 en las estaciones permanentes del Alto Lozoya en primavera. En este caso también hay una correlación significativa entre el EPT y la altitud (LMM, Fig. $3, \mathrm{n}=137, \mathrm{R}_{\mathrm{m}}{ }^{2}=0.14, \mathrm{R}_{\mathrm{c}}{ }^{2}=0.47, p<0.05$ ). La estación más baja (LOZ1090) es la que presenta un peor EPT medio (12.3). En esta estación, mientras que en el IASPT se observa una cierta variabilidad interanual con años relativamente buenos y otros malos, en el caso del EPT los valores son sistemáticamente los más bajos del Alto Lozoya (Fig. 3). Por el contrario, la estación AGU1215 es la que menor variabilidad interanual presenta en el IASPT, y es precisamente en la que se obtiene el mayor EPT (máximo 24, media 19.7). En el caso de los arroyos temporales el EPT medio es de 11.1.

\section{Otras cuencas de la Sierra de Guadarrama}

La variabilidad asociada a las nuevas estaciones de la cara sur es más desconocida al haber menos datos anuales disponibles ( 3 años frente a 13 del Alto Lozoya, Tabla 3). Los resultados también indican un excelente estado de conservación en general al evaluar arroyos permanentes en primavera. De hecho, en el período 2014-2017 en la cuenca del Manzanares y del Guadarrama se obtuvieron 180 y 173 puntos del IBMWP respectivamente, frente a los 175 puntos en la cuenca del Lozoya. Respecto a las métricas IASPT y EPT, en la cuenca del Guadarrama se observan valores algo menores a los del Lozoya. Así, en la del Guadarrama se obtuvo entre 2014-2017 un IASPT medio de 6.28 y un EPT medio de 15.8 (frente a 6.42 y 17.0 respectivamente en la del Lozoya). Sin embargo, en la del Manzanares se aprecia un claro descenso en ambas métricas hasta 6.07 y 14.0 en el IASPT y EPT respectivamente.
Cuando se analiza la correlación entre los diferentes índices biológicos en todos los muestreos de primavera realizados $(\mathrm{n}=165)$, se constata una buena correlación entre el IBMWP y el IASPT (LMM, $\mathrm{R}_{\mathrm{m}}^{2}=0.03, \mathrm{R}_{\mathrm{c}}{ }^{2}=0.82, p<$ $0.0001)$, entre el IBMWP y el EPT (LMM, $\mathrm{R}_{\mathrm{m}}^{2}=$ $\left.0.43, \mathrm{R}_{\mathrm{c}}^{2}=0.87, p<0.0001\right)$, así como entre el IASPT y el EPT (LMM, $\mathrm{R}_{\mathrm{m}}{ }^{2}=0.38, \mathrm{R}_{\mathrm{c}}{ }^{2}=0.55$, $p<0.0001)$. En términos del promedio de estos índices para cada estación, las correlaciones entre ellos se mantienen, pero además es posible encontrar: a) una correlación entre la media del IBMWP y la pendiente media de la cuenca (Slope_Med, $\mathrm{R}^{2}=0.20, p<0.05$ ); $\mathrm{y}$ b) una correlación entre el EPT y la altitud de la estación (Cota_Min, $\mathrm{R}^{2}=0.19, p<0.05$ ).

\section{Cuencas fluviales y relación con los índices biológicos}

Los primeros 4 componentes del PCA explican un $68.5 \%$ de la variabilidad observada en las estaciones de muestreo (Tabla 4 y Fig. 4). El primer componente explica el $24.7 \%$ de la varianza y se asocia principalmente a la altitud media de la cuenca (Cota_Med) y, en su extremo negativo, a la presencia de prados de ladera o fondo de valle (Pastos_Bajos) y la presencia de robledales en la cuenca (Q_Pyrenaica). De esta manera, las estaciones situadas a menor altitud se sitúan en la parte negativa del eje y las situadas a mayor altitud en la parte positiva. En las estaciones situadas a menor altitud hay mayor probabilidad de que parte de la cuenca esté ocupada por los pastos de ladera, de fondo de valle o por el piso de melojar situado por debajo de los pinares de la Sierra. El segundo componente representa un $17.8 \%$ de la varianza total, y está relacionado con la presencia de zonas sin vegetación en la cuenca (Roquedo), y diferencia las cuencas con dominancia de pino albar ( $\mathrm{P} \_$sylvestris) frente a las que tienen otro tipo de pinares (Otros_Pinus) o la presencia de otro arbolado (Otros_Arb). Este componente diferencia claramente las estaciones situadas en La Pedriza, una zona del parque nacional que se caracteriza por tener porcentualmente grandes superficies de roquedo, por la presencia de otros tipos de pinar (Pinus nigra) e incluso superficies relativamente importantes de 
Tabla 3. Resultados del IBMWP, RCE, IASPT y EPT en primavera en otros ríos de la Sierra de Guadarrama. Results of IBMWP, $E Q R, I A S P T$ and EPT during spring in other rivers of the Sierra de Guadarrama.

\begin{tabular}{|c|c|c|c|c|c|c|c|}
\hline Curso Fluvial & Estación & Índice & 穴 & 륨 & 号 & 귬 & $\begin{array}{l}\bar{\pi} \\
\text { 밈 }\end{array}$ \\
\hline \multirow[t]{4}{*}{ Del Mediano } & MED1155 & IBMWP & & & 149 & 166 & 157.5 \\
\hline & & RCE & & & 0.83 & 0.92 & 0.9 \\
\hline & & IASPT & & & 6.8 & 5.9 & 6.3 \\
\hline & & EPT & & & 13 & 14 & 13.5 \\
\hline \multirow[t]{4}{*}{ Majadillas } & MAJ1040 & IBMWP & & & 98 & 119 & 108.5 \\
\hline & & RCE & & & 0.54 & 0.66 & 0.6 \\
\hline & & IASPT & & & 5.8 & 5.7 & 5.7 \\
\hline & & EPT & & & 5 & 8 & 6.5 \\
\hline \multirow[t]{12}{*}{ Manzanares } & MAN1210 & IBMWP & 192 & 184 & 169 & 190 & 183.8 \\
\hline & & RCE & 1.07 & 1.02 & 0.94 & 1.06 & 1.0 \\
\hline & & IASPT & 6.2 & 6.1 & 6.0 & 6.1 & 6.1 \\
\hline & & EPT & 15 & 15 & 14 & 18 & 15.5 \\
\hline & MAN1076 & IBMWP & 197 & 200 & 173 & 177 & 186.8 \\
\hline & & RCE & 1.09 & 1.11 & 0.96 & 0.98 & 1.0 \\
\hline & & IASPT & 5.8 & 5.9 & 5.8 & 5.9 & 5.8 \\
\hline & & EPT & 15 & 12 & 14 & 12 & 13.3 \\
\hline & MAN0965 & IBMWP & 152 & 133 & 120 & 135 & 135.0 \\
\hline & & RCE & 0.84 & 0.74 & 0.67 & 0.75 & 0.8 \\
\hline & & IASPT & 6.1 & 6.6 & 6.0 & 6.8 & 6.4 \\
\hline & & EPT & 12 & 9 & 10 & 11 & 10.5 \\
\hline \multirow[t]{8}{*}{ Navacerrada } & NVC1408 & IBMWP & 181 & & 165 & 178 & 174.7 \\
\hline & & RCE & 1.01 & & 0.92 & 0.99 & 1.0 \\
\hline & & IASPT & 5.8 & & 5.9 & 5.6 & 5.8 \\
\hline & & EPT & 15 & & 16 & 12 & 14.3 \\
\hline & NVC1330 & IBMWP & 208 & & 198 & 160 & 188.7 \\
\hline & & RCE & 1.16 & & 1.10 & 0.89 & 1.0 \\
\hline & & IASPT & 6.1 & & 6.2 & 6.4 & 6.2 \\
\hline & & EPT & 18 & & 17 & 13 & 16.0 \\
\hline \multirow[t]{4}{*}{ Navalmedio } & NAV1436 & IBMWP & 215 & & 197 & 191 & 201.0 \\
\hline & & RCE & 1.19 & & 1.09 & 1.06 & 1.1 \\
\hline & & IASPT & 6.5 & & 6.2 & 6.0 & 6.2 \\
\hline & & EPT & 18 & & 16 & 17 & 17.0 \\
\hline \multirow[t]{8}{*}{ Venta } & VEN1380 & IBMWP & 212 & & 175 & 179 & 188.7 \\
\hline & & RCE & 1.18 & & 0.97 & 0.99 & 1.0 \\
\hline & & IASPT & 6.8 & & 6.3 & 7.2 & 6.8 \\
\hline & & EPT & 20 & & 12 & 17 & 16.3 \\
\hline & VEN1270 & IBMWP & 160 & & 144 & 147 & 150.3 \\
\hline & & RCE & 0.89 & & 0.80 & 0.82 & 0.8 \\
\hline & & IASPT & 5.7 & & 6.0 & 5.9 & 5.9 \\
\hline & & EPT & 17 & & 12 & 13 & 14.0 \\
\hline \multirow[t]{4}{*}{ Canencia } & CAN1180 & IBMWP & 199 & & 175 & 170 & 181.3 \\
\hline & & RCE & 1.11 & & 0.97 & 0.94 & 1.0 \\
\hline & & IASPT & 6.0 & & 6.5 & 5.5 & 6.0 \\
\hline & & EPT & 16 & & 16 & 14 & 15.3 \\
\hline
\end{tabular}


otras especies arbóreas (Cupressus arizonica). Por ello en la parte positiva del eje se sitúan las estaciones del río Manzanares y, muy particularmente, el arroyo de Majadillas, que reflejan las características propias de La Pedriza. En el tercer componente (varianza explicada $13.4 \%$ ) sigue siendo relevante la presencia de los pinares característicos de esta sierra (P_sylvestris) frente a la vegetación de mayor altitud, constituida por pastos psicroxerófilos (Pastos_cumbre) y áreas de piornal/enebral. En la parte negativa del eje suelen situarse las estaciones con grandes extensiones de pinares (como el caso del arroyo de La Venta), mientras que en la parte positiva aparecen tanto estaciones sin grandes extensiones de este pinar (por ejemplo, las del río Manzanares) como estaciones con una presencia comparativamente mayor de pastos psicroxerófilos, piornales y enebrales. El cuarto componente (no se muestra en la figura 4) explica un $12.6 \%$ adicional y está controlado fundamentalmente por la presencia de prados húmedos de altura (Cervunal) así como con la orientación N-S de las cuencas.

El análisis del RDA (Tabla 5 y Fig. 5) indica que las puntuaciones medias de los índices biológicos (mean.IBMWP, mean.IASTP, mean.EPT) se correlacionan fuertemente con los tres ejes $(p<$ 0.001 ), consiguiendo el RDA explicar un $66 \%$ de

Tabla 4. Análisis de Componentes Principales (PCA). Porcentaje de la varianza explicada por cada componente y la acumulada en los primeros cuatro. Factores de carga de las variables sobre los primeros 4 ejes del PCA. Principal Component Analysis (PCA). Percentage of the variance explained by each component and the cumulative variance in the first four. Loading factors of the variables on the first 4 axes of the PCA.

\begin{tabular}{lllll}
\hline & PCA1 & PCA2 & PCA3 & PCA4 \\
$\begin{array}{llll}\text { Porcentaje de varianza } \\
\text { explicada }\end{array}$ & $24.7 \%$ & $17.8 \%$ & $13.4 \%$ & $12.6 \%$ \\
Porcentaje acumulado & $24.7 \%$ & $42.5 \%$ & $55.9 \%$ & $68.5 \%$ \\
\hline
\end{tabular}

Correlación de las variables

\begin{tabular}{lcccccccc} 
& PCA1 & cos2 & PCA2 & cos2 & PCA3 & cos2 & PCA4 & cos2 \\
\hline Area & -0.40 & 0.16 & 0.06 & 0.00 & 0.57 & 0.33 & -0.39 & 0.15 \\
Cota_Min & 0.62 & 0.39 & -0.50 & 0.25 & -0.37 & 0.13 & 0.02 & 0.00 \\
Cota_Max & 0.56 & 0.31 & -0.14 & 0.02 & 0.54 & 0.29 & -0.35 & 0.12 \\
Cota_Med & 0.90 & 0.81 & -0.32 & 0.10 & 0.14 & 0.02 & 0.05 & 0.00 \\
Slope_Med & 0.60 & 0.36 & 0.48 & 0.23 & -0.36 & 0.13 & -0.15 & 0.02 \\
northness & -0.08 & 0.01 & -0.24 & 0.06 & 0.02 & 0.00 & 0.86 & 0.73 \\
eastness & -0.12 & 0.01 & -0.18 & 0.03 & 0.17 & 0.03 & 0.61 & 0.37 \\
Otros_Arb & -0.06 & 0.00 & 0.72 & 0.52 & -0.13 & 0.02 & 0.39 & 0.15 \\
Humedales & 0.33 & 0.11 & -0.28 & 0.08 & -0.19 & 0.04 & 0.04 & 0.00 \\
Matorral & -0.32 & 0.11 & 0.58 & 0.34 & -0.25 & 0.06 & 0.23 & 0.05 \\
Pastos_cumbre & 0.60 & 0.36 & 0.09 & 0.01 & 0.65 & 0.42 & -0.02 & 0.00 \\
Pastos_bajos & -0.80 & 0.64 & -0.09 & 0.01 & 0.38 & 0.15 & -0.11 & 0.01 \\
P_sylvestris & -0.23 & 0.06 & -0.60 & 0.36 & -0.61 & 0.37 & -0.31 & 0.10 \\
Otros_Pinus & 0.16 & 0.03 & 0.92 & 0.84 & 0.17 & 0.03 & 0.05 & 0.00 \\
Piornal_enebral & 0.53 & 0.28 & -0.27 & 0.07 & 0.63 & 0.39 & 0.25 & 0.06 \\
Cervunal & -0.01 & 0.00 & -0.14 & 0.02 & 0.15 & 0.02 & 0.71 & 0.51 \\
Q_pyrenaica & -0.79 & 0.63 & -0.15 & 0.02 & 0.25 & 0.06 & 0.07 & 0.01 \\
Roquedo & 0.50 & 0.25 & 0.65 & 0.43 & -0.06 & 0.00 & -0.10 & 0.01 \\
Ribera & -0.42 & 0.18 & 0.06 & 0.00 & 0.26 & 0.07 & -0.29 & 0.08 \\
\hline
\end{tabular}


la variabilidad total observada. El modelo en su conjunto permite describir significativamente (test de permutación, $p<0.05$ ) la relación entre las variables explicativas y las de respuesta. No obstante, sólo el primer eje (RDA1) aparece como significativo tras el test de permutación, recogiendo el $91 \%$ de la variabilidad restringida. En este primer eje, los impactos se sitúan claramente en la parte derecha del eje, junto con las variables de cuenca con la clara excepción de la altitud de la estación de muestreo. Así, de acuerdo con el RDA la altitud de la estación de muestreo favorece índices biológicos más altos: a mayor altitud (Cota_Min), mayor puntuación de los índices. Por otro lado, el RDA también muestra que los impactos influyen negativamente sobre los índices biológicos. Parece que el factor más determinante es la temporalidad, tal como ya se apuntaba en la comparación del IBMWP entre las estaciones de tipo permanente y de tipo temporal. A continuación, la ganadería parece ser un impacto también importante. Esto quedaría también reflejado por el hecho de que una mayor cobertura de pastos de fondo de valle (mayoritariamente dedicados a este uso) genera una alteración incluso mayor sobre los índices biológicos. Por último, la alteración de la ribera, la presencia de áreas edificadas o la existencia de áreas recreativas (asociadas o no con actividades de baño) parecen compartir un efecto algo menor sobre el descenso de los índices. En este sentido también es reseñable que la cobertura de roquedo sin vegetación ejerce una respuesta similar en los índices, y es que hasta cierto punto vendría a ser un análogo natural de una zona urbana sin vegetación de ribera.

\section{DISCUSIÓN}

\section{Estado Ecológico de los cursos fluviales del PNSG}

En términos generales, los cursos de agua del PNSG y su entorno inmediato están en un estado ecológico bueno o muy bueno. Esta situación ha permanecido estable en la última década, o incluso con una ligera tendencia a la mejoría. En el PNSG hay numerosas estaciones que presentan un índice mayor que el de referencia, es decir, un estado ecológico aún mejor. Aunque este hecho
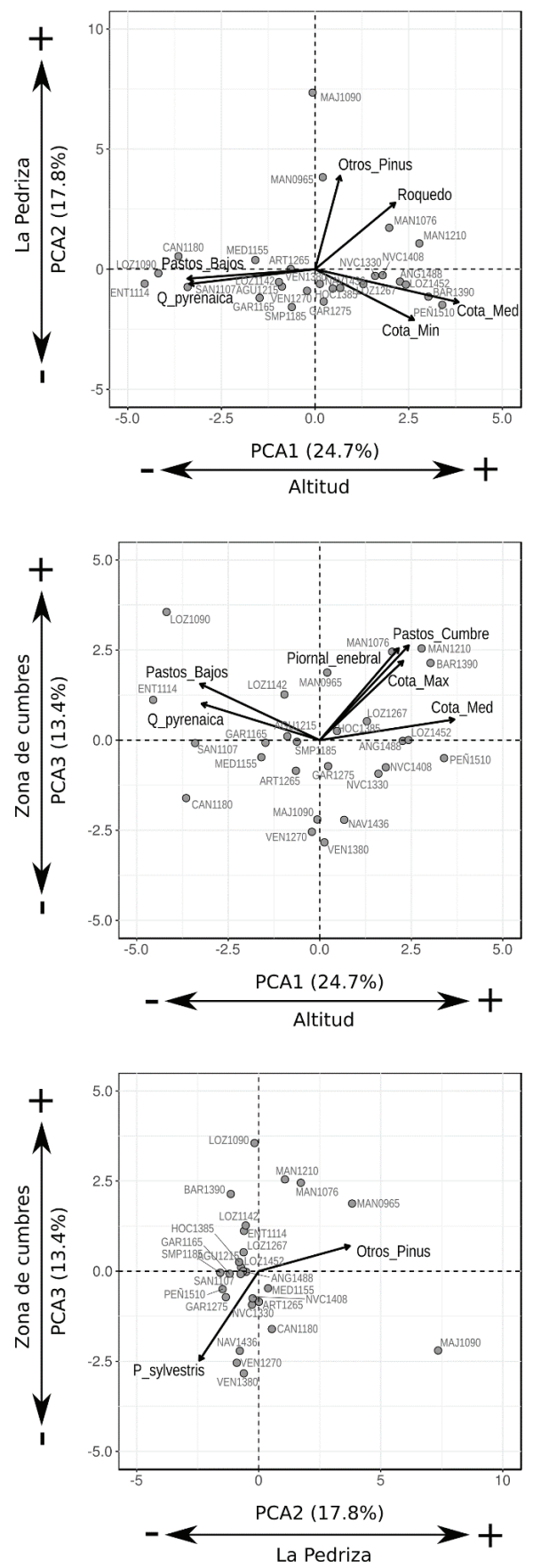

Figura 4. PCA biplot mostrando el primer, segundo y tercer componente con las variables morfométricas de la cuenca y los diferentes tipos de vegetación (sólo se han representado las variables con $\operatorname{Cos}^{2}>0.6$ ). Los puntos son las distintas estaciones de muestreo. PCA biplot showing the first, second and third components with the morphometric variables of the catchment and the different vegetation typologies (only the variables with $\mathrm{Cos}^{2}>0.6$ have been represented). Points are the different sampling stations. 
Tabla 5. Análisis de Redundancia (RDA). Porcentaje de la varianza explicada y la acumulada por los ejes del RDA. Factores de carga de las variables explicativas sobre los ejes del modelo (solo el primero es significativo). Redundancy Analysis (RDA). Percentage of the variance explained by each $R D A$ axis and the cumulative one. Loading factors of the explicative variables on the model axes (only the first one is significative).

\begin{tabular}{cccc}
\hline & RDA1 & RDA2 & RDA3 \\
Porcentaje de varianza explicada & $61.3 \%$ & $3.8 \%$ & $1.6 \%$ \\
Porcentaje acumulado & $61.3 \%$ & $65.0 \%$ & $66.6 \%$ \\
\hline Variables restringidas & & & \\
& & & \\
\hline Temporal & RDA1 & RDA2 & RDA3 \\
Urbano & 0.84 & -0.26 & -0.23 \\
Ganado & 0.16 & 0.10 & -0.30 \\
Ribera_Alt & 0.39 & 0.36 & -0.56 \\
A_Recret & 0.21 & 0.31 & -0.22 \\
Cota_Min & 0.17 & -0.02 & 0.23 \\
Roquedo & -0.68 & -0.20 & -0.21 \\
Pastos_bajos & 0.18 & 0.27 & 0.64 \\
\hline
\end{tabular}

podría estar en relación con las excepcionales cualidades de un parque nacional, en realidad la superación de los valores de referencia es un hecho relativamente frecuente (Couto-Mendoza et al., 2015). Para el tipo R-T11, entre $2006 \mathrm{y}$ 2014 en toda la cuenca del Tajo el valor de referencia del IBMWP se superó en el $19.4 \%$ de los casos, cifra muy similar al $18.4 \%$ observado en la Sierra de Guadarrama. Sin embargo, la media de la cuenca del Tajo fue de 131 frente a 174 en la Sierra de Guadarrama. Es decir, lo que realmente diferencia al PNSG es que la gran mayoría de ríos están bastante bien conservados, más que el tener una diversidad de macroinvertebrados fluviales particularmente notable. En comparación con otros parques nacionales, por ejemplo en el de Ordesa y Monte Perdido (PNOMP) se encuentran valores más bajos del IBMWP que en el PNSG, pero con RCE relativamente elevados e incluso ocasionalmente por encima del nivel de referencia (Valladolid et al., 2015). También aparece algún punto con valores deficientes.

En cuanto a la mejor época de muestreo, a la vista del RDA y de la serie histórica de datos se concluye que la mejor época de muestreo para la Sierra de Guadarrama es la primavera tardía, salvo que se quiera evaluar una actividad claramente ligada al verano cómo pudiera ser el caso del baño. Esta recomendación sólo debería aplicarse al PNSG, ya que por ejemplo en el PNOMP los valores máximos se encuentran en verano, en vez de en primavera, aunque probablemente sea por un muestreo excesivamente prematuro (mayo) para los Pirineos (Valladolid et al., 2015). Por su parte, Álvarez-Cabria (2010) encuentra los mayores valores del IBMWP en otoño para los ríos de Cantabria, y se ha sugerido (Álvarez-Cabria et al., 2010) que probablemente esta sea la mejor época para la monitorización de ríos templados atlánticos y similares.

\section{Factores que influyen en los índices biológicos}

La ordenación de las estaciones de muestreo con el PCA muestra que los factores más relevantes se corresponden con un gradiente altitudinal (reflejado también en los diferentes pisos de vegetación), así como con la pertenencia a La Pedriza, un área del parque nacional notablemente diferente al resto y caracterizado por grandes extensiones de roquedo y una vegetación singular. El RDA muestra que estas diferencias entre las estaciones influyen en la puntuación de los índices biológicos, al igual que los principales impactos detectados en la Sierra de Guadarrama. De esta manera, la utilización de los índices biológicos en el sistema de seguimiento del PNSG parece proporcionar una herramienta efectiva en la detección de impactos sobre los cursos fluviales.

Dentro del patrón general de mejorar la puntuación de los índices con la altitud, es destacable que se pueden obtener valores muy elevados de los índices biológicos en estaciones situadas a relativamente baja altura siempre y cuando los impactos en la cuenca sean despreciables. Esto puede ser el resultado de que las estaciones de media ladera o incluso de fondo de valle puedan reunir unas condiciones que propicien una mayor diversidad de macroinvertebrados acuáticos: mayor diversidad de hábitats fluviales, mayor desarrollo aluvial, un bosque de ribera mejor estructurado, etc (Bordallo López \& Casado Sancho, 2012). Por otra parte, la configuración dendrítica de la red de drenaje implica que 


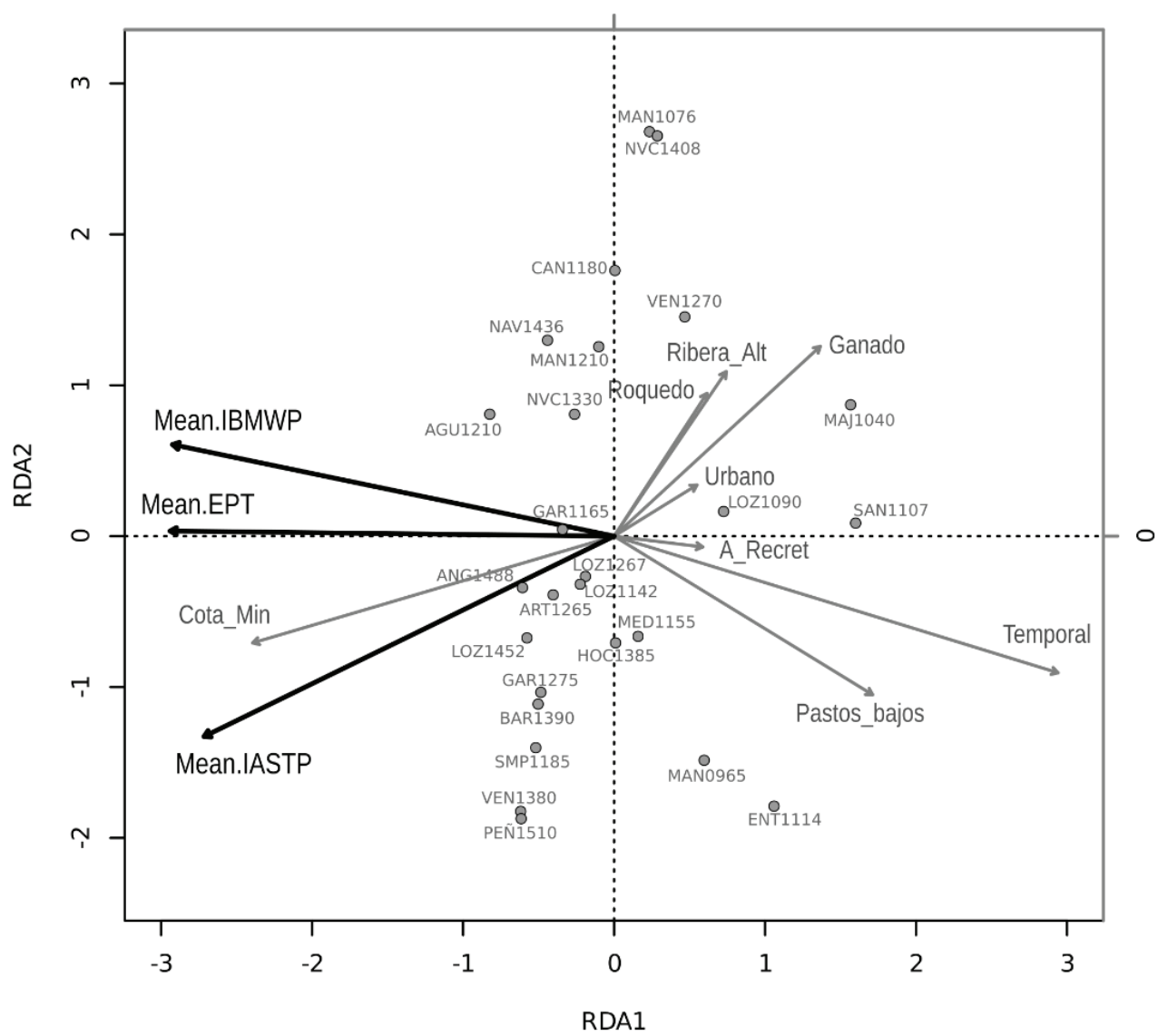

Figura 5. Análisis de redundancia (RDA), triplot con los índices biológicos (negro), las características de cuenca más relevantes e impactos (gris), y las estaciones de muestreo (gris claro). Redundancy analysis (RDA), triplot with the biological indices (black), the most relevant basin characteristics and impacts (grey), and the sampling stations (light grey).

los tramos de cabecera permanezcan más aislados y predominen en ellos los factores locales frente a una mayor contribución de los factores regionales en tramos más bajos (Brown \& Swan, 2010). Estos últimos tramos están más conectados e influenciados por las comunidades situadas aguas arriba. Además, la riqueza de especies en un sistema dendrítico es mayor a niveles intermedios de la estructura ramificada y la diversidad de especies aumentan en las confluencias de las ramas (Altermatt, 2013), es decir, fuera de las zonas de cabecera.

Sin embargo, frente a la mayor potencialidad para albergar más diversidad de macroinvertebrados, en las estaciones más bajas de la Sierra de Guadarrama suelen presentarse una acumulación de diversos impactos: 1) las estaciones tempora- les suelen estar en altitudes bajas, frecuentemente asociada a la infiltración en el contacto con las calizas del fondo de valle del Lozoya; 2) el impacto ganadero cobra mayor importancia, al concentrarse en pastos de ladera y fondo de valle; 3) la mayor parte de las áreas recreativas y la actividad de baño se realizan en zonas donde el río tiene ya mayores dimensiones; 4) las zonas más bajas suelen tener las riberas más degradadas. El descenso de los valores del IBMWP desde las áreas de cabecera y aquellas con mayor influencia antrópica son frecuentes en zonas de montaña (Tobes et al., 2016).

Por otro lado, algunas de las estaciones a mayor altitud no son las que mayor puntuación alcanzan (Fig. 2). A partir de cierta altitud el bosque de ribera va desapareciendo progresiva- 
mente, a lo que contribuyen razones climáticas (especialmente la temperatura), así como el tratamiento forestal que históricamente se ha hecho de estos pinares. En el caso de la restricción climática, en otro parque nacional también se ha descrito la disminución del número de familias en las estaciones más elevadas (Valladolid et al., 2015) por causas naturales asociadas a una menor presencia de restos vegetales, menor producción primaria, períodos más cortos para el crecimiento, etc. En el río Manzanares (PNSG), también se ha observado un menor número de especies y menor densidad de macroinvertebrados en su tramo más elevado (1713 m s.n.m) que en su tramo medio (1020 m s.n.m.; Gutiérrez Teira, 2003). En definitiva, la temperatura es un factor limitante para el desarrollo de muchas especies en cotas más elevadas, y controla la composición de la comunidad.

En el caso del tratamiento de los pinares, durante décadas se ha favorecido la presencia de pinar frente a los bosques de ribera de planifolios para la explotación comercial. Aunque no está claro qué variables ambientales influyen en la descomposición de la materia orgánica de origen terrestre en la Sierra de Guadarrama (Casado et al., 2015), el tipo de hojarasca pueden determinar importantes cambios en la comunidad y funcionamiento de estos ríos (Casas et al., 2013). De hecho, en PEÑ1510 (con relativamente bajo IBMWP) se observan valores muy altos de IASPT y EPT, lo que indica que apenas hay contaminación. Por todo ello, los mayores valores de los índices biológicos encontrados en la Sierra de Guadarrama se encuentren en torno a las altitudes medias (1200-1400 m s.n.m.). A partir de esta cota los arroyos permanentes de menor entidad (Peñalara y Barondillo) sin impactos en su cuenca, presentan un IBMWP menor que los arroyos similares de cotas más bajas.

\section{Aplicación de las clases de calidad al PNSG}

El Plan Director de Parques Nacionales (MAGRAMA, 2016), contempla el seguimiento del estado ecológico de los cursos fluviales mediante los parámetros contemplados en la DMA. Sin embargo, es necesario señalar que los umbrales que se utilizan oficialmente (MAGRAMA, 2015) han sido desarrollados para cuencas de más de $10 \mathrm{~km}^{2}$ y para recoger todo el rango posible de degradación de los ríos españoles. En las

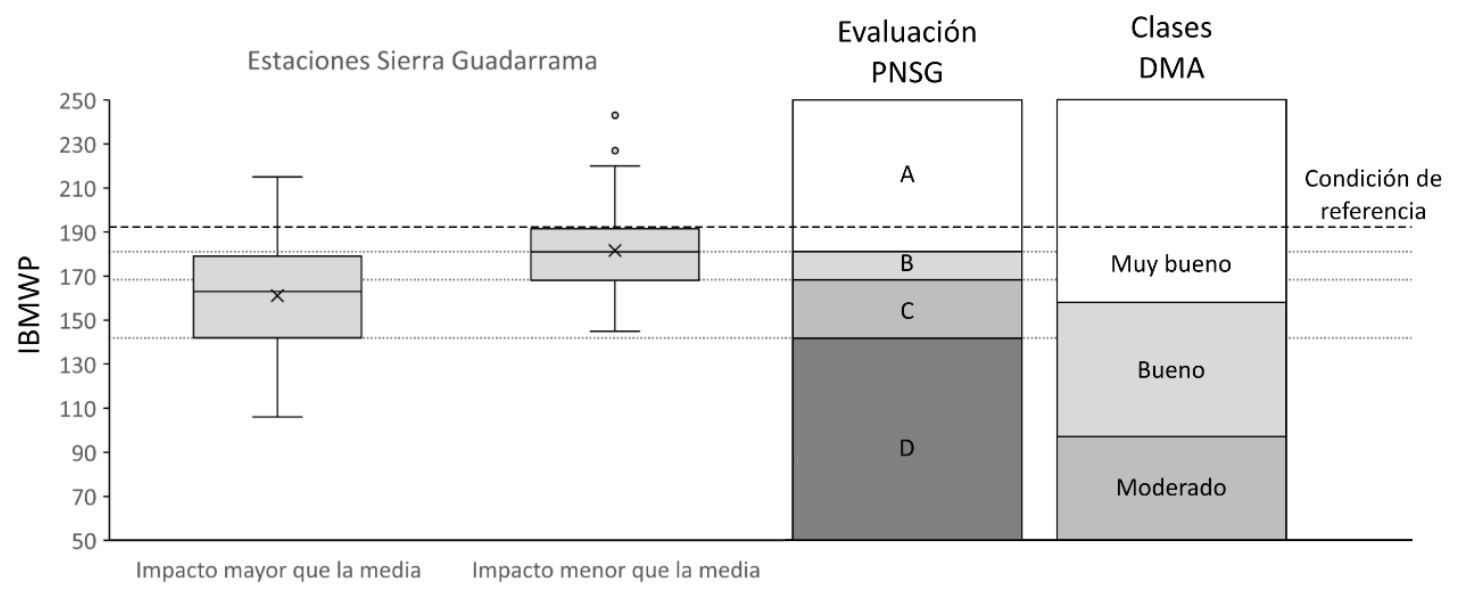

Figura 6. Distribución de los valores del IBMWP en las estaciones con impactos acumulados por encima o por debajo de la media observada en la Sierra de Guadarrama: media, mediana, cuartiles, máximo, mínimo y puntos anómalos. A la derecha se muestran los intervalos correspondientes tanto a las clases de la DMA (R-T11), como al sistema de evaluación y seguimiento del Parque Nacional de la Sierra de Guadarrama (PNSG). Distribution of the IBMWP values in the stations with cumulative impacts above or below the average observed in the Sierra de Guadarrama: mean, median, quartiles, maximum, minimum and anomalous points. On the right are the intervals corresponding to the WFD (R-T11) classes and also the evaluation and monitoring system of the Sierra de Guadarrama National Park (PNSG). 
estaciones estudiadas en la Sierra de Guadarrama, más de un tercio tiene una cuenca inferior a estos $10 \mathrm{~km}^{2}$. Además, a pesar de que en la Sierra de Guadarrama se ha encontrado una respuesta de los índices biológicos a los impactos en la cuenca, esta reducción queda englobada casi siempre en las categorías de buena o muy buena calidad ecológica. Es muy probable que el cambio de clase de calidad ecológica buena a moderada ocurra cuando los impactos sobre determinado río del parque nacional sean ya muy elevados. Como consecuencia de ello, en el marco de la evaluación del estado ecológico de un parque nacional puede ser conveniente establecer unos umbrales de corte diferentes y específicos de los requeridos estrictamente por la DMA.

Para establecer estos valores, se han seleccionado los resultados $(\mathrm{n}=89)$ de los índices biológicos correspondientes a las estaciones permanentes cuyo impacto total es menor de la media del conjunto de estaciones estudiadas, frente a los resultados $(\mathrm{n}=57)$ del resto de estaciones con un impacto por encima de la media (Fig. 6). Como puede observarse, la clasificación oficial (DMA) no permite ofrecer más información sobre el estado ecológico de los ríos del parque nacional más allá de constatar que, en el contexto de ese ecotipo, los ríos de esta sierra están relativamente bien conservados. Para poder tomar decisiones de gestión frente a los impactos $\mathrm{y}$ anticiparse a cambios graves en el parque nacional, se ha establecido otra clasificación de la calidad del estado ecológico según el IBMWP (Fig. 6) para los ríos permanentes basados en esta distribución de los valores observados en las estaciones mejor y peor conservadas:

- El percentil $75 \%$ de las puntuaciones de las estaciones con bajo impacto se sitúa en 192 puntos, prácticamente equivalente a la condición de referencia del ecotipo R-T11 y por tanto se asume también como condición de referencia para el PNSG

- La media de las puntuaciones en las estaciones con bajo impacto se sitúa en 181 puntos. Se asignan a la categoría A las estaciones con una puntuación mayor, y se consideran como ríos naturalizados o sin impactos apreciables.

- El $25 \%$ de las puntuaciones de estaciones con bajo impacto no alcanzan 169 puntos, valor cercano a la media de las estaciones con más impactos (163 puntos). Se asignan a la categoría $\mathrm{B}$ las estaciones con una puntuación entre 169 y 181 puntos, y se consideran como ríos con indicios de impactos.

- El $25 \%$ de las puntuaciones de estaciones con mayores impactos no alcanzan los 142 puntos. Se asignan a la categoría $\mathrm{C}$ las estaciones con una puntuación entre 142 y 168 puntos, y se consideran como ríos en los que los impactos están causando con bastante probabilidad una degradación en el medio fluvial. En estos casos se deberían hacer estudios adicionales para clarificar y cuantificar estos impactos.

- Por debajo de 142 puntos, se establece la categoría $\mathrm{D}$, en la que el río presenta un estado especialmente alterado de acuerdo con los valores esperables para la Sierra de Guadarrama. La realización de estudios adicionales debería considerarse una prioridad en la gestión del Parque nacional.

Se trata de límites de cambio más rigurosos de los empleados para establecer las clases de calidad oficiales (Pujante et al., 2016), pero probablemente mucho más adecuados para detectar de forma temprana alteraciones en los cursos fluviales de un área con la máxima protección medioambiental. Esta clasificación específica para el PNSG debe evaluarse complementariamente con los resultados del IAST y del EPT para determinar el grado de afección por impactos.

En el caso de los ríos temporales, parece que el IBMWP no es tan efectivo por lo que se han propuesto otros índices alternativos o complementarios (IMMi-L o IMMi-t; Munné \& Prat, 2009) Tentativamente, en la Sierra de Guadarrama se puede aplicar una reducción de los límites anteriormente comentados del $10 \%$ en ríos temporales con pozas (intermitentes) y del $40 \%$ en ríos que se secan completamente. Estos porcentajes son equivalentes a la reducción media observada entre los ríos permanentes y ambos tipos de ríos temporales. Por otra parte, el IASPT, si bien no tiene en cuenta la diversidad de los macroinvertebrados, nos da una idea de la calidad de los taxones que habitan estos cursos temporales en la Sierra de Guadarrama, por 1o que puede ser una herramienta para la evaluación de los ríos temporales. 


\section{AGRADECIMIENTOS}

Este estudio se enmarca en el seguimiento del estado ecológico de los ríos y arroyos del Parque Natural de Peñalara (2005-2013) y del Parque Nacional de la Sierra de Guadarrama (2014-2017), financiado por la Dirección General de Medio Ambiente y Sostenibilidad de la Comunidad de Madrid.

\section{BIBLIOGRAFÍA}

ABDI, H. \& L. J. WILLIAMS. 2010. Principal component analysis. Wiley Interdisciplinary Reviews: Computational Statistics, 2 (4): 433-459. DOI: 10.1002/wics.101

ALBA-TERCEDOR, J., P. JÁIMEZ-CUÉLLAR, M. ÁlVAREZ, J. AVILÉS, N. BONADA, J. CASAS, A. MELLADO, M. ORTEGA, I. PARDO, N. PRAT, M. RIERADEVALL, S. ROBLES, C. E. SÁINZ-CANTERO, A. SÁNCHEZ-ORTEGA, M. L. SUÁREZ, M. TORO, M. R. VIDAL-ABARCA, S. VIVAS \& C. ZAMORA-MUÑOZ. 2002. Caracterización del estado ecológico de ríos mediterráneos ibéricos mediante el índice IBMWP (antes BMWP'). Limnetica, 21 (3-4): 175-185.

ALBA-TERCEDOR, J., I. PARDO, N. PRAT \& A. PUJANTE. 2005. Protocolos de Muestreo y Análisis para Invertebrados Bentónicos. Ministerio de Medio Ambiente. Madrid. España.

ALTERMATT, F. 2013. Diversity in riverine metacommunities: a network perspective. Aquatic Ecology, 47 (3): 365-377. DOI: 10.1007/s10452-013-9450-3

ÁLVAREZ-CABRIA, M. 2010. Estudio de la variabilidad espacio-temporal de las comunidades de macroinvertebrados bentónicos en los ecosistemas fluviales de Cantabria: repercusiones para la aplicación de la Directiva Marco del Agua. Ph. D. Thesis. Universidad de Cantabria, Spain.

ÁLVAREZ-CABRIA, M., J. BARQUÍN \& J. A. JUANES. 2010. Spatial and seasonal variability of macro invertebrate metrics: Do macroinvertebrate communities track river health? Ecological Indicators, 10 (2):
370-379. DOI: 10.1016/j.ecolind.2009.06.018 AQEM CONSORTIUM. 2002. Manual for the application of the AQEM system. A comprehensive method to assess European streams using benthic macroinvertebrates, developed for the purpose of the Water Framework Directive. Informe Técnico Version 1.0, $202 p p$.

BARQUÍN, J., F.J. PEÑAS, M. ÁLVAREZ-CABRIA, T. RODRÍGUEZ-CASTILLO, A.M. GONZÁLEZ, E. ESTÉVEZ, M. LEZCANO, F. FERNANDEZ, J. ROJO, A. GARCÍA \& C. ÁLVAREZ. 2015. Optimización de las redes de seguimiento del estado de conservación en ríos de alta montaña. En: Proyectos de investigación en parques nacionales: 2010-2013. OAPN (ed.):35-59. Colección Investigación en la Red de Parques Nacionales. MAGRAMA.

BARTOŃ, K. 2018. MuMIn: Multi-Model Inference. $R$ package version 1.42.1. https:// CRAN.R-project.org/package=MuMIn

BATES, D., M. MÄCHLER, B. BOLKER \& S. WALKER. 2015. Fitting Linear MixedEffects Models Using lme4. Journal of Statistical Software, 67 (1): 1-48. DOI: 10.18637/ jss.v067.i01

BORDALLO LÓPEZ, A. \& C. CASADO SANCHO. 2012. Aproximación al establecimiento de una red de referencia para la implantación de la Directiva Marco del Agua en la Comunidad de Madrid. Limnetica, 31 (1): 1-12.

BROWN, B.L. \& C.M. SWAN. 2010. Dendritic network structure constrains metacommunity properties in riverine ecosystems. Journal of Animal Ecology, 79 (3): 571-580. DOI: 10.1111/j.1365-2656.2010.01668.x

CASADO, C., L. LOSADA \& S. MOLLÁ. 2011. Efecto de las áreas de protección especial en la comunidad de macroinvertebrados de los ríos de la cuenca alta del Manzanares (Sierra de Guadarrama, Madrid). Limnetica, 30 (1): 71-88.

CASADO, C., S. MOLLÁ, J. M. GONZÁlEZ, N. ROBLAS \& E. DESCALS. 2015. Procesado de hojarasca en arroyos del Parque Nacional de la Sierra de Guadarrama (Madrid). Limnetica, 34 (1): 115-134.

CASAS, J. J., A. LARRAÑAGA, M. MENÉN- 
DEZ, J. POZO, A. BASAGUREN, A. MARTÍNEZ, J. PÉREZ, J. M. GONZÁLEZ, S. MOLLÁ, C. CASADO, E. DESCALS, N. ROBLAS, J. A. LÓPEZ-GONZÁLEZ \& J. L. VALENZUELA. 2013. Leaf litter decomposition of native and introduced tree species of contrasting quality in headwater streams: How does the regional setting matter? Science of The Total Environment, 458-460: 197-208. DOI: 10.1016/j.scitotenv.2013.04.004

COMUNIDAD DE MADRID. 2018. Mosaico a escala 1:5.000 de máxima actualidad de ortofotografía del Plan Nacional de Ortofotografía Aérea de la Comunidad de Madrid. http://www.madrid.org/catalogocartografia/ catalog/search/resource/details.page?uuid= \{E7A7EB35-E6F0-458B-A9EC-DF83E68B9 C17\}.

CONFEDERACIÓN HIDROGRÁFICA DEL TAJO. 2018. Resultados obtenidos para la calidad biológica en ríos. Resultados/informes: aguas superficiales - estado/potencial ecológico en ríos. http://www.madrid.org/ catalogocartografia/catalog/search/resource/ details.page?uuid $=\{\mathrm{E} 7 \mathrm{~A} 7 \mathrm{~EB} 35-\mathrm{E} 6 \mathrm{~F} 0-458 \mathrm{~B}-$ A9EC-DF83E68B9C17\}.

COUTO-MENDOZA, M.T., R. VIEIRA-LANERO \& F. COBO. 2015. More complexity does not always mean more accuracy: the case of IBMWP and METI in NW Spain. Ecohydrology, 8 (4): 595-609. DOI: 10.1002/eco.1528

FOX, J. 2005. The R Commander: A Basic Statistics Graphical User Interface to R. Journal of Statistical Software, 14 (9): 1-42.

GUTIÉRREZ TEIRA, B. 2003. Variaciones de las comunidades y poblaciones de macroinvertebrados del tramo alto del río Manzanares a causa de las temperaturas: posibles efectos del cambio. Ph. D. Thesis. Universidad Politécnica de Madrid, Spain.

JÁIMEZ-CUÉLLAR, P., S. VIVAS, N. BONADA, S. ROBLES, A. MELLADO, M. ÁlVAREZ, J. AVILÉS, J. CASAS, M. ORTEGA, I. PARDO, N. PRAT, M. RIERADEVALL, C. E. SÁINZ-CANTERO, A. SÁNCHEZ-ORTEGA, M.L. SUÁREZ, M. TORO, M. R. VIDAL-ABARCA, C. ZAMORA-MUÑOZ \& J. ALBA-TERCEDOR. 2002. Protocolo Guadalmed (PRECE).
Limnetica, 21(3-4): 187-204.

LÊ, S., J. JOSSE \& F. HUSSON. 2008. FactoMineR: an $\mathrm{R}$ package for multivariate analysis. Journal of statistical software, 25 (1): 1-18. DOI: $10.18637 /$ jss.v025.i01

MAGRAMA. 2013. Protocolo de cálculo del indice IBMWP. Código: IBMWP-2013. Ministerio de Medio Ambiente, Medio Rural y Marino. Madrid. España.

MAGRAMA. 2015. Real Decreto 817/2015 por el que se establecen los criterios de seguimiento y evaluación del estado de las aguas superficiales y las normas de calidad ambiental. Boletín Oficial del Estado-BOE 219 (Ministerio de Agricultura, Alimentación y Medio Ambiente): 80582-80677.

MAGRAMA. 2016. Real Decreto 389/2016, de 22 de octubre, por el que se aprueba el Plan Director de la Red de Parques Nacionales. Boletín Oficial del Estado-BOE 257 (Ministerio de Agricultura, Alimentación y Medio Ambiente): 74051-74076.

MUNNÉ, A. \& N. PRAT. 2009. Use of macroinvertebrate-based multimetric indices for water quality evaluation in Spanish Mediterranean rivers: an intercalibration approach with the IBMWP index. Hydrobiologia, 628 (1): 203-225. DOI: 10.1007/s10750-009-9757-1

MUÑOZ MUNICIO, C., T. GIL, P. DE LAS HERAS \& N. GONZÁLEZ. 2004. Memoria del Mapa de Vegetación de la Sierra de Guadarrama (Vertiente madrileña). Centro de Investigaciones Ambientales de la Comunidad de Madrid "Fernando González Bernáldez". Informe Técnico.

NAKAGAWA, S. \& H. SCHIELZETH. 2012. A general and simple method for obtaining $\mathrm{R}^{2}$ from generalized linear mixed-effects models. Methods in Ecology and Evolution, 4 (2): 133-142. DOI: 10.1111/j.2041-210x.2012. 00261.x

OKSANEN, J., F. G. BLANCHET, M. FRIENDLY, R. KINDT, P. LEGENDRE, D. MCGLINN, P. R. MINCHIN, R. B. O'HARA, G. L. SIMPSON, P. SOLYMOS, M. H. H. STEVENS, E. SZOECS \& H. WAGNER. 2018. vegan: Community Ecology Package. https://CRAN.R-project.org/package=vegan

PRAT, N. \& A. MUNNÉ. 2014. Biomonitoreo de 
la calidad del agua en los ríos ibéricos: lecciones aprendidas. Limnetica, 33 (1): 47-64.

PUJANTE, A.M., A. PUIG, E. BARRIOS \& J. RUZA. 2016. Contribución al establecimiento de condiciones de referencia y límites entre clases de estado ecológico en los ríos españoles. Limnetica, 35 (1): 201-218. DOI: 10.23818/limn.35.17

R CORE TEAM. 2017. R: A Language and Environment for Statistical Computing. R Foundation for Statistical Computing. Vienna, Austria.

RUBIO-ROMERO, A. \& I. GRANADOS. 2013. El Alto Lozoya. Seguimiento ecológico de los cursos fluviales. Evaluación y estado de conservación (2002-2013). Centro de Investigación, Seguimiento y Evaluación del Parque Nacional de la Sierra de Guadarrama. Madrid. Informe Técnico, 118pp.

RUIZ-GARCÍA, A. \& M. FERRERAS-ROMERO. 2015. El estado ecológico de las pequeñas cuencas de cabecera en las serranías béticas húmedas (parque natural Los Alcornocales, sur de España) según la Directiva Marco del Agua: ¿su aplicación garantiza la conservación? Animal Biodiversity and Conservation, 38 (1): 59-69.

SÁINZ BARIÁIN, M. 2014. Diversidad, estrategias vitales y filogeografia de especies sensibles al cambio climático. Ph. D. Thesis. Universidad de Granada, Spain

SALMI, T., A. MÄÄTTÄ, P. ANTTILA, T. RUOHO-AIROLA \& T. AMNELL. 2002. Detecting trends of annual values of atmospheric pollutants by the Mann-Kendall test and Sen's slope estimates -the Excel template application MAKESENS. Finnish Meteorological Institute. Helsinki.

TOBES, I., S. GASPAR, J. OSCOZ \& R. MIRANDA. 2016. Diagnosing stream ecosystem integrity in the Ordesa-Vinamala Biosphere Reserve, central Spanish Pyrenees. Journal of Applied Ichthyology, 32 (1): 229-239. DOI: 10.1111/jai.13026

TORO, M., S. ROBLES, I. TEJERO, E. CRIS-
TÓBAL, S. VELASCO, J. R. SÁNCHEZ \& A. PUJANTE. 2009a. Grupo 32. Tipo Ecológico No 11. Ríos de montaña mediterránea silícea. En: Bases ecológicas preliminares para la conservación de los tipos de hábitat de interés comunitario en España. 15. Ministerio de Medio Ambiente, y Medio Rural y Marino. Madrid.

TORO, M., S. ROBLES, I. TEJERO, E. CRISTÓBAL, S. VELASCO, J.R. SÁNCHEZ \& A. PUJANTE. 2009b. Grupo 32. Tipo Ecológico No 27. Ríos de alta montaña. En: Bases ecológicas preliminares para la conservación de los tipos de hábitat de interés comunitario en España. Ministerio de Medio Ambiente, $\mathrm{y}$ Medio Rural y Marino. Madrid.

VALLADOLID, M., M. ARAUZO \& L. JIMÉNEZ. 2015. Estado ecológico de los ríos incluidos dentro del Parque Nacional de Ordesa y Monte Perdido (cuenca del Ebro, Aragón), mediante indicadores de macroinvertebrados. Pirineos. Revista de Ecología de Montaña, 170. DOI: 10.3989/Pirineos.2015.170002

VALLADOLID, M., M. ARAUZO \& J. J. MARTÍNEZ-BASTIDA. 2010. Estado ecológico del río Oja (cuenca del Ebro, La Rioja, España), mediante indicadores de macroinvertebrados. Limnetica, 29 (2): 393-406.

ZAMORA-MUÑOZ, C., M. SÁINZ-BARIÁIN, C. MÚRRIA, N. BONADA, C.E. SÁINZ-CANTERO, M. A. GONZÁLEZ, J. ALBA-TERCEDOR \& J. M. TIERNO DE FIGUEROA. 2011. Diversidad, estrategias vitales y filogeografía de especies sensibles al cambio climático: Tricópteros en el Parque Nacional de Sierra Nevada. En: Proyectos de investigación en parques nacionales: 2008-2011. OAPN (ed.):355-385. Colección Investigación en la Red de Parques Nacionales. MAGRAMA.

ZUUR, A. F., E. N. IENO \& C. S. ELPHICK. 2010. A protocol for data exploration to avoid common statistical problems. Methods in Ecology and Evolution, 1 (1): 3-14. DOI: 10.1111/j.2041-210X.2009.00001.x

Con el apoyo de:
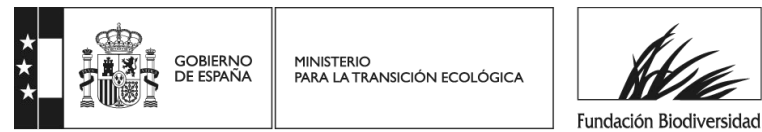